\title{
RESEARCH
}

Open Access

\section{Signature changes in gut microbiome are associated with increased susceptibility to HIV-1 infection in MSM}

Yue Chen ${ }^{1 *}$ D , Huang Lin ${ }^{2,3}$, Mariah Cole ${ }^{1,4}$, Alison Morris ${ }^{5}$, Jeremy Martinson ${ }^{1}$, Heather Mckay ${ }^{6}$, Matthew Mimiaga ${ }^{7}$, Joseph Margolick ${ }^{8}$, Adam Fitch ${ }^{4}$, Barbara Methe ${ }^{4}$, Vatsala Rangachar Srinivas ${ }^{1}$, Shyamal Peddada ${ }^{2,3}$ and Charles R. Rinaldo ${ }^{1}$

\begin{abstract}
Background: Men who have sex with men (MSM) have been disproportionately affected by HIV-1 since the beginning of the AIDS pandemic, particularly in the USA and Europe. Compared to men who have sex with women (MSW), MSM have a distinct fecal microbiome regardless of HIV-1 infection. However, it is unclear whether the MSM-associated gut microbiome affects the susceptibility and progression of HIV-1 infection. We studied fecal microbiome profiles, short-chain fatty acids, and blood plasma inflammatory cytokines of $109 \mathrm{HIV}-1$ seroconverters (SC) from the early, 1984-1985 phase of the HIV-1 pandemic in the Multicenter AIDS Cohort Study (MACS) before and after HIV-1 infection compared to 156 HIV-1-negative MACS MSM (negative controls [NC]).
\end{abstract}

Results: We found that family Succinivibrionaceae, S24-7, Mogibacteriaceae, Coriobacteriaceae, and Erysipelotrichaceae were significantly higher $(p<0.05)$, whereas Odoribacteraceae, Verucomicrobiaceae, Bacteroidaceae, Barnesiellaceae, and Rikenellaceae were significantly lower $(p<0.05)$, in SC before HIV-1 infection compared to NC. At the species level, Prevotella stercorea, Eubacterium biforme, and Collinsella aerofaciens were significantly higher $(p<0.05)$, and Eubacterium dolichum, Desulfovibrio D168, Alistipes onderdonkii, Ruminococcus torques, Bacteroides fragilis, Bacteroides caccae, Alistipes putredinis, Akkermansia muciniphila, Bacteroides uniformis, and Bacteroides ovatus were significantly lower $(p<0.05)$ in SC before HIV-1 infection compared to NC. After HIV-1 infection, family Prevotellaceae and Victivallaceae and species Bacteroides fragilis and Eubacterium cylindroides were significantly higher $(p<0.05)$ in SC who developed AIDS within 5 years compared to the SC who were AIDS free for more than 10 years without antiretroviral therapy (ART). In addition, family Victivallaceae and species Prevotella stercorea, Coprococcus eutactus, and Butyrivibrio crossotus were significantly higher $(p<0.05)$ and Gemmiger formicilis and Blautia obeum were significantly lower $(p<0.05)$ after HIV-1 infection in SC who developed AIDS within 5-10 years compared to the SC who were AIDS-free for more than 10 years without ART. Furthermore, plasma inflammatory cytokine levels of sCD14, sCD163, interleukin 6, and lipopolysaccharide binding protein were significantly higher in SC with $p<0.05$ before HIV-1 infection compared to NC.

\footnotetext{
*Correspondence: cheny@pitt.edu

Yue Chen and Huang Lin are the co-first authors of the study.

Shyamal Peddada and Charles R. Rinaldo are the co-senior authors of this

study.

'Department of Infectious Diseases and Microbiology, University of

Pittsburgh Graduate School of Public Health, Pittsburgh, PA, USA

Full list of author information is available at the end of the article
} 
Conclusions: Our results suggest that pathogenic changes in the gut microbiome were present in MSM several months prior to infection with HIV-1 in the early phase of the AIDS pandemic in the USA. This was associated with increased inflammatory biomarkers in the blood and risk for development of AIDS.

Keywords: Fecal microbiome, HIV seroconversion, AIDS, MSM (men who have sex with men), Inflammation

\section{Background}

The human gastrointestinal (GI) tract possesses gutassociated lymphoid tissue (GALT) which orchestrates host-microbe symbiosis [1]. The gut microbiome also has an important role in maintaining GALT homeostasis and preventing microbial translocation and systemic immune activation [2,3]. Regardless of the route of HIV-1 infection, the virus quickly moves to GALT and rapidly replicates in part due to the high level of residential activated $\mathrm{CD} 4^{+} \mathrm{T}$ cells. This results in a rapid and severe depletion of $\mathrm{CD}_{4}^{+} \mathrm{T}$ cells, immune activation, and gut microbiome dysbiosis. These pathogenic changes can lead to microbial translocation, systemic immune activation, and disease progression [4-6].

There are numerous factors that can affect gut microbiome composition, such as genetics, diet, age, antibiotic usage, sex behaviors, and disease status [7-9]. In people with HIV-1 infection (PWH), a number of crosssectional fecal microbiome studies have shown characteristics of a fecal bacterial community with lower alpha diversity and increased abundance of Proteobacteria and Prevotella with decreased abundance of Bacteroidetes, Firmicutes, and Erysipelotrichaceae in ART-naïv PWH [5, 6, 10-14]. However, few studies of gut microbiome changes in early HIV-1 infection without ART have been reported due to the challenges of recruitment of such individuals and the advent of pre-exposure prophylaxis. Gori et al. [15] reported that in 57 asymptomatic, antiretroviral treatment (ART)-naive HIV-1-infected Italian men and women, there is an increased abundance of Pseudomonas aeruginosa and Candida albicans, which is associated with increased microbial translocation, higher viral loads, and lower $\mathrm{CD} 4^{+} \mathrm{T}$ cell percentages, and decreased abundance of Lactobacilli and Bifidobacteria. Rocafort et al. [16] showed in 49 Mozambican men and women that acute HIV-1 infection led to transient, non-HIV-1-specific changes in gut bacterial richness and composition. Currently, there is no information directly comparing the gut microbiome prior to and soon after HIV-1 infection.

In the USA, men who have sex with men (MSM) have been disproportionately affected by HIV-1 since the early 1980s [17]. Recent studies [18-20] have shown that MSM have a distinct fecal microbiome compared to men who have sex with women (MSW), regardless of HIV-1 infection. In the early years of the HIV-1 pandemic, the greatest risk factor for acquiring HIV-1 in
MSM was being the recipient in unprotected analreceptive intercourse $[21,22]$. We hypothesized that the unfavorable interplay of the host gut microbiome and the gut-associated immune system could be a predisposing factor for HIV-1 infection in MSM. Moreover, we further hypothesized the changes of the gut microbiome following HIV-1 infection have an unfavorable impact on HIV-1 disease progression. The answers to these questions could have important implications in the clinical management of acute HIV-1-infected individuals.

In this study, we analyzed longitudinal fecal and blood plasma samples that were obtained from MSM in the Multicenter AIDS Cohort Study (MACS) early in the HIV-1/AIDS pandemic. We explored the changes of the gut microbiome and fecal bacterial product short-chain fatty acids (SCFA) several months before and after documented HIV-1 infection in MSM as well as in HIV-1negative MSM controls to evaluate the gut microbiome in relation to HIV-1 acquisition and early risk factors for developing AIDS.

\section{Methods}

\section{Stool and plasma samples}

Stool and plasma samples were obtained from the specimen cryorepositories of the MACS (http:// aidscohortstudy.org/). The MACS is a prospective cohort study of HIV-1 infection in MSM established in 1983 at 4 sites (Baltimore, Maryland/Washington, DC; Chicago, Illinois; Los Angeles, California; Pittsburgh, Pennsylvania) [23], that has recently joined with the Women's Interagency HIV Study (WIHS) to form the MACS-WIHS Combined Cohort Study. MACS participants have been studied at semiannual clinic visits with standardized interviews, physical examinations, and phlebotomy for laboratory testing, with storage of plasma and serum and viable peripheral blood mononuclear cells. The study was conducted with institutional review board approval from all participating institutions. During the early phase of the HIV-1 pandemic (19841985), MACS participants were instructed to provide stool, urine, semen, and oral wash samples at each clinical research visit, which have been preserved at $-80^{\circ} \mathrm{C}$ without additives or preservatives. Stools were sampled in $20 \mathrm{ml}$ screw-capped glass vials at home and delivered to the clinic within one day by the participants. Enrollment and clinical research of the MACS participants began April 1, 1984, with clinical research visits at 6- 
month intervals thereafter. During that early period, a number of the MACS participants were infected by HIV-1. HIV-1 seroconversion was determined by enzyme-linked immunosorbent assay and confirmed by Western blot with the participants' serum samples. The HIV-1 infection date was estimated as the midpoint between the last seronegative and first seropositive clinic research visits [23-25]. In the current study, we examined stool and plasma samples from the clinic research visits flanking the estimated HIV-1 infection time point from 109 HIV-1 seroconverters, and from 156 HIV-1 uninfected MSM controls collected during the same time period. The plasma HIV-1 loads were determined retrospectively by Roche quantitative RT-PCR with a detection limit of $300 \mathrm{cp} / \mathrm{ml}$ or Roche Ultra-sensitive quantitative RT-PCR with a detection limit of $40 \mathrm{cp} / \mathrm{ml}$. The paired samples spanned approximately 6 months, which included an estimated 3 months prior to and 3 months after HIV-1 infection. HIV-1 seroconverters and controls were matched by study centers. All samples were obtained in 1984-1985. Of the 109 HIV-1 seroconverters, 32 developed AIDS [26] within 5 years after seroconversion, 31 developed AIDS within 5-10 years, and 46 were AIDS-free for more than 10 years after seroconversion without ART.

\section{Profiling microbial populations by sequencing of the variable region of the $16 \mathrm{~S}$ rRNA gene}

Fecal DNA was extracted from the stool samples using the PowerSoil DNA Extraction Kit (MO BIO Laboratories, Carlsbad, CA, USA). The V4 variable region in the 16S rRNA gene was PCR-amplified with the universal primers: 515F 5'-(GTG CCA GCM GCC GCG GTA A)3' and 806R 5'-(GGA CTA CHV GGG TWT CTA AT)3 ' [27]. DNA concentrations were measured using Qubit 4. Fluorometer (Thermo Fisher Scientific, Waltham, MA, USA). Amplicons were cleaned, pooled, and sequenced on an Illumina MiSeq platform according to the manufacturer's specifications to generate paired-end reads. The datasets generated and/or analyzed during the current study are available in the GitHub repository (https://github.com/FrederickHuangLin/GutMicrobiome-and-HIV-Infection).

\section{S rRNA gene sequence analysis}

The resulting $16 \mathrm{~S}$ rRNA gene sequence data were processed using QIIME2 (version 2019.10.0). The raw sequences were first demultiplexed, and then denoised to remove noisy reads, dereplicated to reduce repetition, and clustered into amplicon sequence variants (ASVs) using the DADA2 algorithm (https://pubmed.ncbi.nlm. nih.gov/27214047/). The observed counts of ASVs were organized into a large matrix referred to as the feature table, where columns represent samples and rows represent ASVs. No ASV was removed based on its observed abundance. The taxonomic composition of bacterial communities was investigated by classifying sequences to the latest reference database (Silva 132 99\% OTUs [full-length, seven-level taxonomy, release 132]) using a Naive Bayes classifier.

\section{Fecal short-chain fatty acid (SCFA) measurement}

To determine the concentration of SCFA in the stool samples, $50-100 \mathrm{mg}$ fecal matter were aliquoted from every stool sample and sent to the University of Pittsburgh Health Sciences Metabolomics and Lipidomics Core in the Department of Pharmacology \& Chemical Biology. The fecal acetate, butyrate, propionate, and valerate levels were measured by stable isotope dilution liquid chromatography mass spectrometry [28].

\section{Measurement of plasma inflammatory cytokines}

For the heparinized plasma samples, the inflammatory cytokines soluble CD14 (sCD14), soluble scavenger receptor CD163 (sCD163), C-reactive protein (CRP), interferon $\gamma$-induced protein $10 \quad$ (IP-10), and lipopolysaccharide-binding protein (LBP) were measured with the Luminex xMAP platform (Luminex, Northbrook, IL, USA), according to the manufacturer's instructions. The data were collected and analyzed using a BioPlex 200 apparatus and BioPlex Manager Software (Bio-Rad, Hercules, CA, USA). In addition, the inflammatory cytokine interleukin 6 (IL-6) was measured in the plasma samples by ELISA using a commercial ELISA kit (R\&D, Minneapolis, MN, USA) following the manufacturer's instructions.

\section{Statistical analyses}

\section{Analysis of gut microbiota composition}

The differential abundance (DA) analysis was performed using Analysis of Compositions of Microbiomes with Bias Correction (ANCOM-BC) [29]. The challenge of DA analysis is the compositional nature of microbiome data due to sampling and sequencing depth (the number of reads assigned to an ASV must be interpreted relative to the total number of reads obtained for that sample). ANCOM-BC properly accounts for the compositional nature of the microbiome data by suitably estimating and eliminating the bias introduced by differences in sampling fractions in the observed counts. This methodology uses relative abundances to infer absolute abundances while controlling false discovery rate (FDR) and deals with excess zero by incorporating the ANCOM-II procedure [30]. Microbial absolute abundances were compared between study groups (seroconverters (SC) vs. negative controls $(\mathrm{NC})$ ) and different visits (last seronegative visit (visit 1) vs. first seropositive visit (visit 2)) adjusting for age. Furthermore, microbial abundances 
were also compared in SC based on their years to AIDS diagnosis [26]. Unadjusted $p$-values were used to visualize the results due to a lack of power. Adjusted $p$ values by the Benjamini-Hochberg procedure [31] were also provided in the corresponding supplementary tables.

\section{Analysis of gut microbiota's gene richness and diversity}

Both alpha (within-sample) and beta (between-sample) diversities were computed using $\mathrm{R}$ microbiome package [32] (Tools for microbiome analysis in R. Version 2.1.24. URL: http://microbiome.github.com/microbiome.) on $\mathrm{R}$ Studio (Version 1.2.5033). The calculations of alpha and beta diversities were based on rarefied data (subsample taxa without replacement based on the $90 \%$ of minimum library size) since the differential library sizes can have a significant impact on alpha and beta diversities [33].

Inter and intra-study group alpha diversities were evaluated at different visits. Two alpha diversity measurements were investigated: (1) observed number of species or species richness, and (2) Shannon diversity index, which accounts for richness (i.e., the number of species), evenness (i.e., a measure to quantify how equal the microbial abundance is numerical), and divergence (i.e., the variance of species). The corresponding $p$-value was obtained by applying Kruskal-Wallis rank sum test between groups. The $\log _{2}$ transformed $\mathrm{CD} 4^{+} / \mathrm{CD}^{+}$ratio was used as a measure of the immune status of HIV-1infected participants. Although individually $\mathrm{CD}^{+}$and $\mathrm{CD}^{+}$are important, it is their relative value that is biologically more meaningful because these are compositional. Since statistically, log-ratios provide better normal approximation than the ratio, analyses were performed using $\log _{2}$ transformed $\mathrm{CD} 4^{+} / \mathrm{CD}^{+}$ratio. Also note that log-ratio transformation is typically implemented in analyzing compositional data [34]. The relationship between Shannon diversity index and $\log _{2}\left(\mathrm{CD}^{+} / \mathrm{CD}^{+}\right)$as well as the relationship between Shannon diversity index and virus load (only for SC at visit $2, \log _{10}$ transformed) were also surveyed. The $p$ value was obtained by fitting a linear regression model between Shannon diversity index, as the outcome variable, and the explanatory variables $\left(\log _{2}\left(\mathrm{CD} 4^{+} / \mathrm{CD} 8^{+}\right)\right.$or virus load) and, age stratified by the study group.

Bray-Curtis dissimilarity was selected as the beta diversity measure in this study. $P$-value was obtained by Permutational Multivariate Analysis of Variance (PERMANOVA) (https://onlinelibrary.wiley.com/doi/ full/10.1111/j.1442-9993.2001.01070.pp.x). Since a significant PERMANOVA test implies that the observed differences (in multivariate space) are either due to different spatial medians or the heterogeneity of dispersions of different groups, a Permutational Analysis of Multivariate Dispersion (PERMDISP, https:// onlinelibrary.wiley.com/doi/pdf/10.1111/j.1541-0420.2 005.00440.x ? casa_token $=\mathrm{C} 1 \mathrm{nt}$ h hAeSVsAAAAA: fy NhMhnZ1V 5 _CeR0E S b LEuvefJ D t 5 h 5 E 6 rFNoFTDE9sUknMBTvqC0FBXMwfT8o2obpKf-VQW4 r1SNK6p) test is also performed to confirm the leading effect. An ordination is a popular approach for visualizing and exploring microbial community composition. We projected the samples using principal coordinates (PCoA) plots in the context of Bray-Curtis dissimilarity to visualize between-sample distance.

\section{Analysis of SCFA and inflammatory cytokine difference between groups}

SCFA levels were investigated between study groups at different visits (NC vs. SC at visit 1 ; NC vs. $\mathrm{SC}$ at visit 2 ). The $p$-value was obtained by applying Kruskal-Wallis rank sum test between groups. The relationship between SCFA and $\log _{2}\left(\mathrm{CD}^{+} / \mathrm{CD}^{+}\right)$as well as the relationship between SCFA and virus load (only for SC at visit 2, $\log _{10}$ transformed) were also surveyed. The $p$-value was obtained by fitting a linear regression model between SCFA, as the outcome variable, and the explanatory variables $\left(\log _{2}\left(\mathrm{CD}^{+} / \mathrm{CD}^{+}\right)\right.$or virus load) and age, stratified by the study group. Similar analyses were performed for the inflammatory cytokines.

\section{Results}

Study participants and clinical samples

For this study, the SC and NC were selected and matched from the 4 MACS sites (Baltimore/Wash DC, Chicago, Los Angeles, and Pittsburgh) (Table 1). The HIV-1 infection events in SC were retrospectively reconfirmed by RT-PCR with cryopreserved plasma, with HIV-1 antibody and RNA being negative at visit 1 and both being positive at visit 2 with approximately 6month intervals. The HIV-1 negativity of $\mathrm{NC}$ was confirmed by negative plasma HIV-1 antibody results from both visits. All study participants were self-defined MSM. The SC did not receive ART due to the unavailability of effective antiretroviral drugs in the early years of the HIV-1 pandemic. Stool specimens were obtained from MACS participants beginning April 1, 1984, in order to investigate these specimens for potential viruses that caused AIDS. With the confirmation of HIV-1 as the etiologic agent of AIDS in May, 1984, and prior to the modern resurgence of research on the microbiome, the MACS began to discontinue collection of stools and ended this practice in late 1985. Moreover, not all MACS participants were able to supply stool specimens at each study visit. Thus, within that time, there were 35 SC and $77 \mathrm{NC}$ with plasma and stool samples from both visits 1 and 2, $52 \mathrm{SC}$ and $79 \mathrm{NC}$ only with visit 1 samples, and $22 \mathrm{SC}$ only with visit 2 samples. Compared to NC, SC were younger $(p=0.02)$, but had comparable 
Table 1 Characteristics of study participants

\begin{tabular}{|c|c|c|c|}
\hline & HIV seroconverters $(N=109)$ & HIV-negative controls $(N=156)$ & $P$-value \\
\hline \multicolumn{4}{|l|}{ Data type } \\
\hline Samples from visits $1 \& 2$ available & $35(32 \%)$ & 77 (49\%) & \\
\hline Samples from visit 1 available & $52(48 \%)$ & $79(51 \%)$ & \\
\hline Samples from visit 2 available & $22(20 \%)$ & $0(0 \%)$ & $<0.01$ \\
\hline \multicolumn{4}{|l|}{ Time to AIDS after HIV-1 infection } \\
\hline$<5$ years & $32(29)$ & NA & \\
\hline $5-10$ years & $31(28)$ & NA & \\
\hline$>10$ years & $46(42)$ & NA & NE \\
\hline \multicolumn{4}{|l|}{ Time period for paired data (days) } \\
\hline Min & 141 & 77 & \\
\hline Max & 547 & 230 & \\
\hline Mean (sd) & $204.37 \pm 63.62$ & $182.60 \pm 24.49$ & 0.03 \\
\hline \multicolumn{4}{|l|}{ Age } \\
\hline Min & 19 & 21 & \\
\hline Max & 82 & 80 & \\
\hline Mean (sd) & $37.12 \pm 13.84$ & $42.42 \pm 16.64$ & 0.02 \\
\hline \multicolumn{4}{|l|}{ Location } \\
\hline Baltimore & $22(20 \%)$ & $48(31 \%)$ & \\
\hline Chicago & $32(29 \%)$ & $39(25 \%)$ & \\
\hline Pittsburgh & $20(18 \%)$ & $27(17 \%)$ & \\
\hline Los Angeles & $35(32 \%)$ & $42(27 \%)$ & 0.28 \\
\hline
\end{tabular}

NE not evaluable

$\mathrm{CD}^{+} \mathrm{T}$ cell counts, $\mathrm{CD}^{+} \mathrm{T}$ cell counts, and $\mathrm{CD}^{+} /$ $\mathrm{CD}^{+}$ratios at their pre-HIV-1 infection visit (visit 1 ). $\mathrm{SC}$ had detectable plasma HIV-1 loads, lower $\mathrm{CD}^{+}{ }^{+} \mathrm{T}$ cell counts, and lower $\mathrm{CD} 4^{+} / \mathrm{CD}^{+}$ratio at the postHIV-1 infection, seroconversion visit (visit 2) compared to NC (Fig. 1).

\section{S rRNA gene sequencing}

A total of 19,500,780 sequence reads were generated for the 377 stool samples, with an average of 51,726 (range 7-126,903) reads per stool sample (Supplementary Figure S1). In the per-base sequence quality plot, the quality of the initial bases appears to be high for both forward and reverse reads, and the quality of the reverse reads appears to drop off around position 170 similar to all Illumina sequencing data.

\section{Fecal microbiome diversities}

\section{Alpha diversity}

Microbial alpha diversity measures microbial diversity within a sample. Two alpha diversity indices (observed species and Shannon diversity index) were compared at the family level between groups using the standard linear regression method where the $p$-value was adjusted for age. As shown in Fig. 2, there were no differences in observed species and Shannon diversity index between NC and $\mathrm{SC}$ at visit 1 (Fig. 2A). However, at visit 2, there were significant decreases of alpha diversity in SC compared to $\mathrm{NC}$ for both observed species $(p=0.008)$ and Shannon diversity index $(p=0.015)$. Furthermore, compared to $\mathrm{NC}$, there was a significant loss of Shannon diversity index (value at visit 2 minus value at visit 1 ) in $\mathrm{SC}(p=0.03)$ (Fig. $2 \mathrm{C})$. These results suggest that HIV-1 infection reduced the within-sample bacterial diversity.

\section{Correlation analysis of fecal bacterial alpha diversity with $\mathrm{CD} 4^{+} / \mathrm{CD} 8^{+} \mathrm{T}$ cell ratio and HIV-1 load}

The peripheral blood $\mathrm{CD}^{+} / \mathrm{CD}^{+} \mathrm{T}$ lymphocyte ratio is often recognized as a quantitative outcome that reflects the critical role of both $\mathrm{CD}^{+}$and $\mathrm{CD}^{+} \mathrm{T}$ cells in HIV1 pathogenesis or disease progression [35]. To explore the role of the gut microbiome in HIV pathogenesis, linear regression analysis was performed to reveal the relationship between Shannon diversity index of fecal microbiome and the $\log _{2}$ ratio of $\mathrm{CD}^{+} \mathrm{T}$ cells and $\mathrm{CD}^{+} \mathrm{T}$ cells in peripheral blood mononuclear cells collected at the same stool-collection visits. Interestingly, there was no statistically significant correlation between 


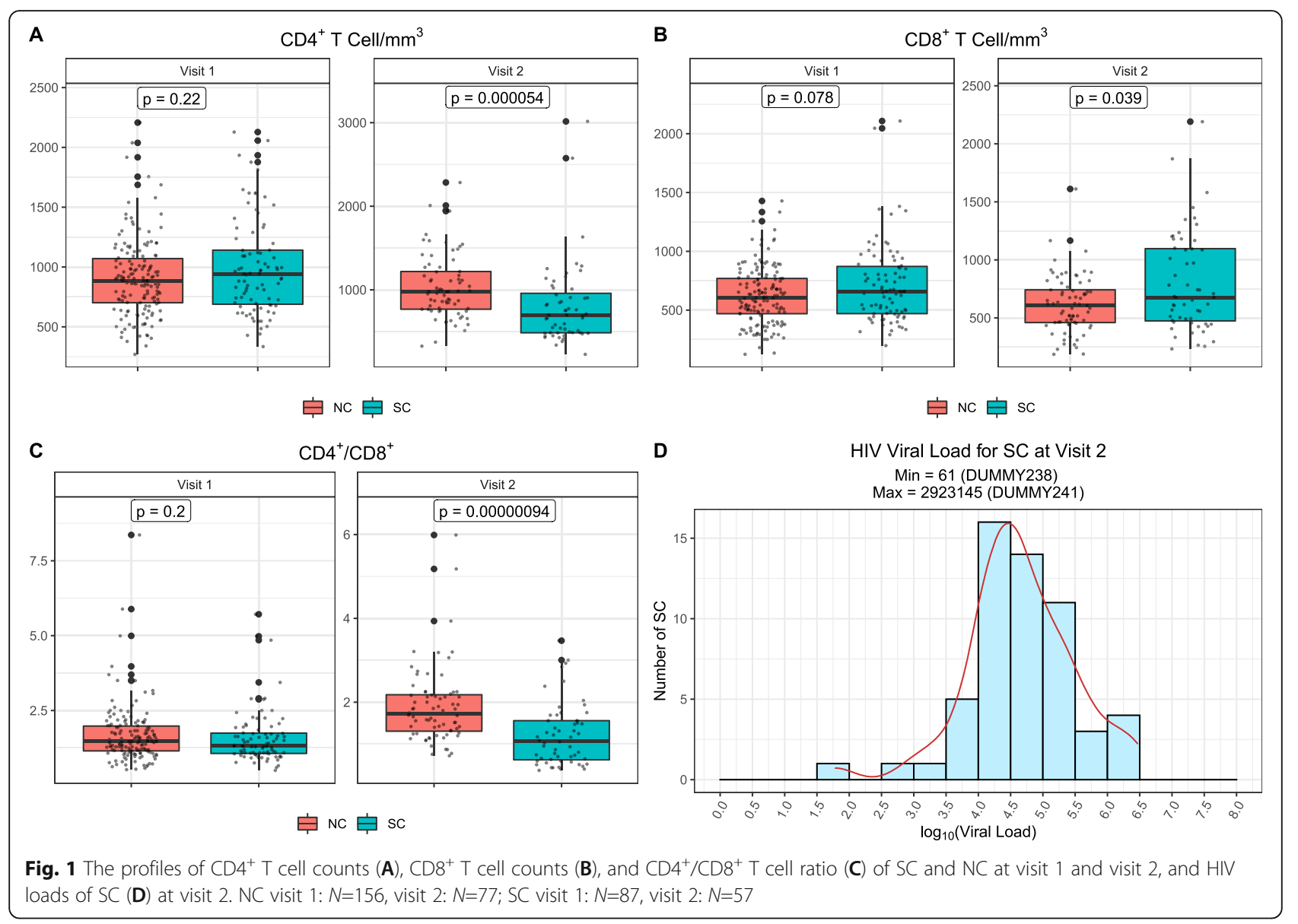

alpha diversity and $\mathrm{CD} 4^{+} / \mathrm{CD} 8^{+}$ratios at visit 1 or visit 2 in both $\mathrm{NC}$ and SC (Supplementary Figure S2A and S2B). In addition, no statistically significant correlation was detected between alpha diversity and HIV-1 loads at visit 2 in SC (Supplementary Figure S3).

\section{Beta diversity}

Bacterial beta diversity shows the difference in taxonomic abundance profiles across different groups of samples. In NC or SC, there was no significant difference of beta diversity between visit 1 samples and visit 2 samples based on PERMANOVA test (Fig. 3A, B). Furthermore, PERMANOVA test reveals a trend of separation, but not statistically significant difference, between spatial medians of beta diversity between NC and SC at visit 1 (Fig. 3C). However, comparing visit 2 samples between SC and NC, a statistically significant difference was detected in beta diversity by PERMANOVA test ( $p$ $=0.044)$; the PERMDISP test $(p=0.21)$ suggested homogeneity of dispersion and the difference in beta diversity relies on their different spatial medians (Fig. 3D). It is possible that HIV-1 infection led to the changes in the taxonomic abundance profiles of microbial communities in these MSMs.

\section{Fecal bacterial compositions}

Despite certain variations among the groups and visits, there were no significant differences in the taxonomic composition of the fecal microbiome at the phylum level between SC and $\mathrm{NC}$ at visit 1 and visit 2 (Supplementary Figure S4). For a deeper understanding of fecal bacterial changes at a lower level of the phylogenic tree, analyses were also performed at the family, genus, and species levels. Compared to $\mathrm{NC}$ at visit 1, 5 bacterial families (Succinivibrionaceae, S24-7, Mogibacteriaceae, Coriobacteriaceae, and Erysipelotrichaceae) were significantly higher in mean abundance (log fold change [LFC, natural $\log$ ] ranges from 0.43 to $1, p<0.05)$ and the mean abundance of 5 bacterial families (Odoribacteraceae, Verrucomicrobiaceae, Bacteroidaceae, Barnesiellaceae, and Rikenellaceae) were significantly lower (LFC ranges from -1.21 to $-0.52, p<0.05$ ) in SC at visit 1 (Fig. 4A, Supplementary Table 1A). Similarly, two families (S24-7 and Coriobacteriaceae) were significantly higher in mean abundance (LFC ranges from 0.83 to $0.87, p<0.05$ ) and 6 families (Pasteurellaceae, Odoribacteraceae, Veillonellaceae, Porphyromonadaceae, Bacteroidaceae, and Barnesiellaceae) were significantly lower mean abundance (LFC ranges from -1.17 to $-0.44, p<0.05$ ) in SC compared to $\mathrm{NC}$ at visit 2 (Fig. 4B, Supplementary Table 1B). Compared to SC visit 1, two families 


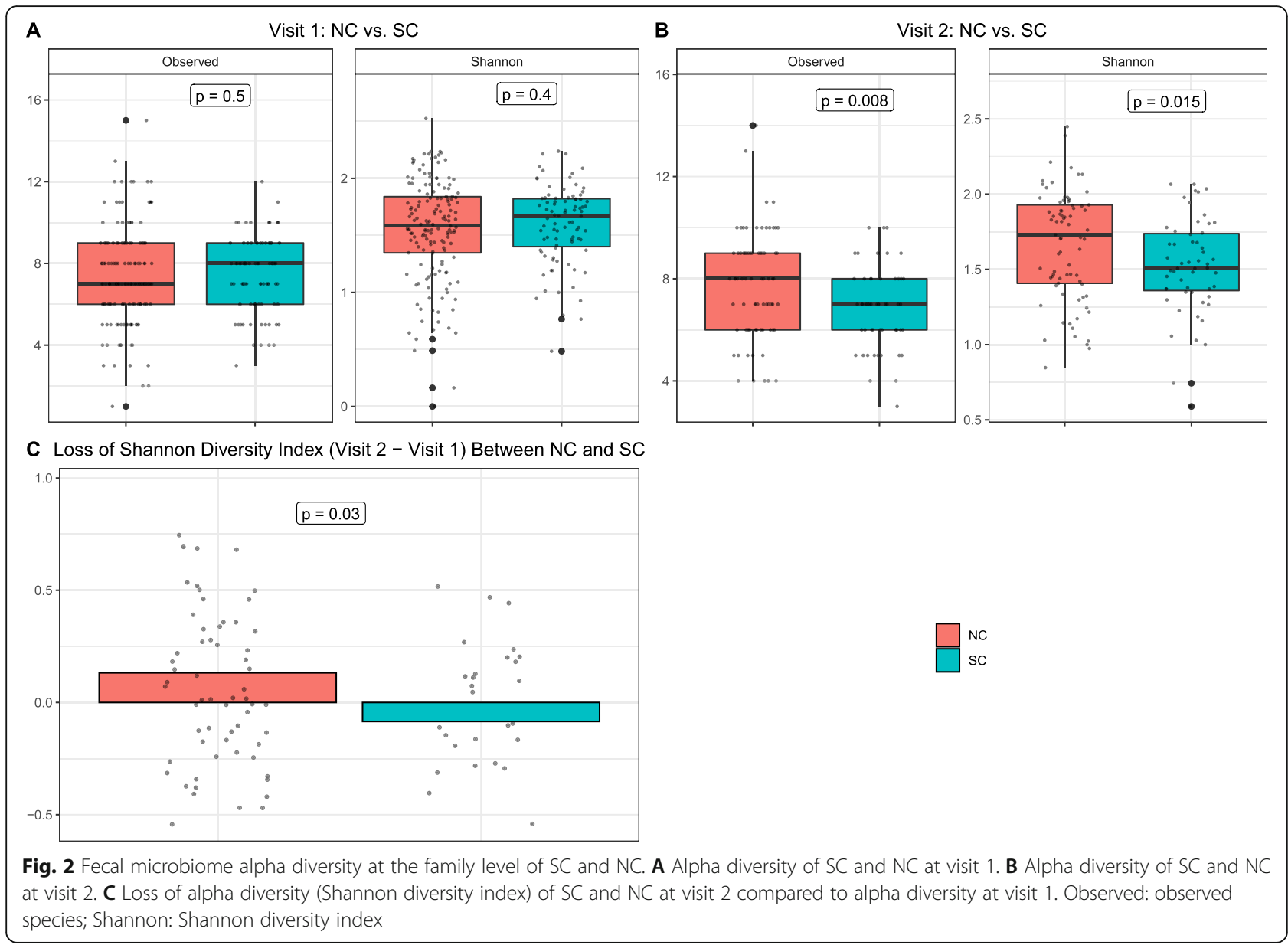

(Tissierellaceae and Enterococcaceae) were significantly higher in mean abundance (LFC ranges from 0.50 to $0.56, p<0.05$ ) and one family (Coriobacteriaceae) was significantly lower in mean abundance (LFC $=-0.71, p<0.05)$ at SC visit 2 after HIV-1 seroconversion (Fig. 4C, Supplementary Table 1C).

At the genus level, compared to NC at visit 1, 17 bacterial genera (Catenibacterium, Mogibacterium, Prevotella, Succinivibrio, Butyrivibrio, Peptococcus, Bulleidia, Desulfovibrio, Eubacterium, Oribacterium, Phascolarctobacterium, Collinsella, Methanobrevibacter, 02d06, Slackia, Anaerovibrio, and p-75a5) were significantly higher in the mean abundance (LFC ranges from 0.34 to $1.69, p<0.05)$ and 4 bacterial taxa (Bilophila, Akkermansia, Bacteroides, and Alistipes) were significantly lower in the mean abundance (LFC ranges from -1.18 to $-0.64, p<0.05$ ) in $\mathrm{SC}$ at visit 1 (Supplementary figure $5 \mathrm{~A}$, Supplementary Table 2A). Similarly, 7 genera (Slackia, Mogibacterium, Catenibacterium, Collinsella, Bulleidia, Eubacterium, and Ruminococcus) were significantly higher in the mean abundance (LFC ranges from 0.63 to $1.11, p<0.05$ ) and 5 genera (Butyricicoccus, Anaerostipes, Odoribacter, Parabacteroides, and Alistipes) were significantly lower in their mean abundance (LFC ranges from -0.94 to $-0.64, p<0.05$ ) in SC compared to $\mathrm{NC}$ at visit 2 (Supplementary figure $5 \mathrm{~B}$,
Supplementary Table 2B). Compared to SC visit 1, three genera (Paraprevotella, Methanosphaera, and Defluviitalea) were significantly higher in their mean abundance (LFC ranges from 0.34 to $0.84, p<0.05$ ), and one genus (Collinsella) was significantly lower (LFC $=-0.78, p<0.05)$ at $\mathrm{SC}$ visit 2 after HIV-1 seroconversion (Supplementary figure 5C, Supplementary Table 2C).

At the species level, compared to $\mathrm{NC}$ at visit 1, three bacterial species (Prevotella Stercorea, Eubacterium Biforme, and Collinsella Aerofaciens) were significantly higher in the mean abundance (log fold change [LFC, natural $\log$ ] ranges from 0.55 to $1.49, p<0.05$ ) and 10 bacterial species (Eubacterium Dolichum, Desulfovibrio D168, Alistipes Onderdonkii, Ruminococcus Torques, Bacteroides Fragilis, Bacteroides Caccae, Alistipes Putredinis, Akkermansia Muciniphila, Bacteroides Uniformis, and Bacteroides Ovatus) were significantly lower in the mean abundance (LFC ranges from -1.16 to $-0.36, p<$ 0.05 ) in SC at visit 1 (Fig. 5A, Supplementary Table 3A). Similarly, 5 bacterial species (Eubacterium biforme, Collinsella aerofaciens, Bulleidia p-1630-c5, Dorea formicigenerans, and Ruminococcus gnavus) were significantly higher in the mean abundance (LFC ranges from 0.61 to 


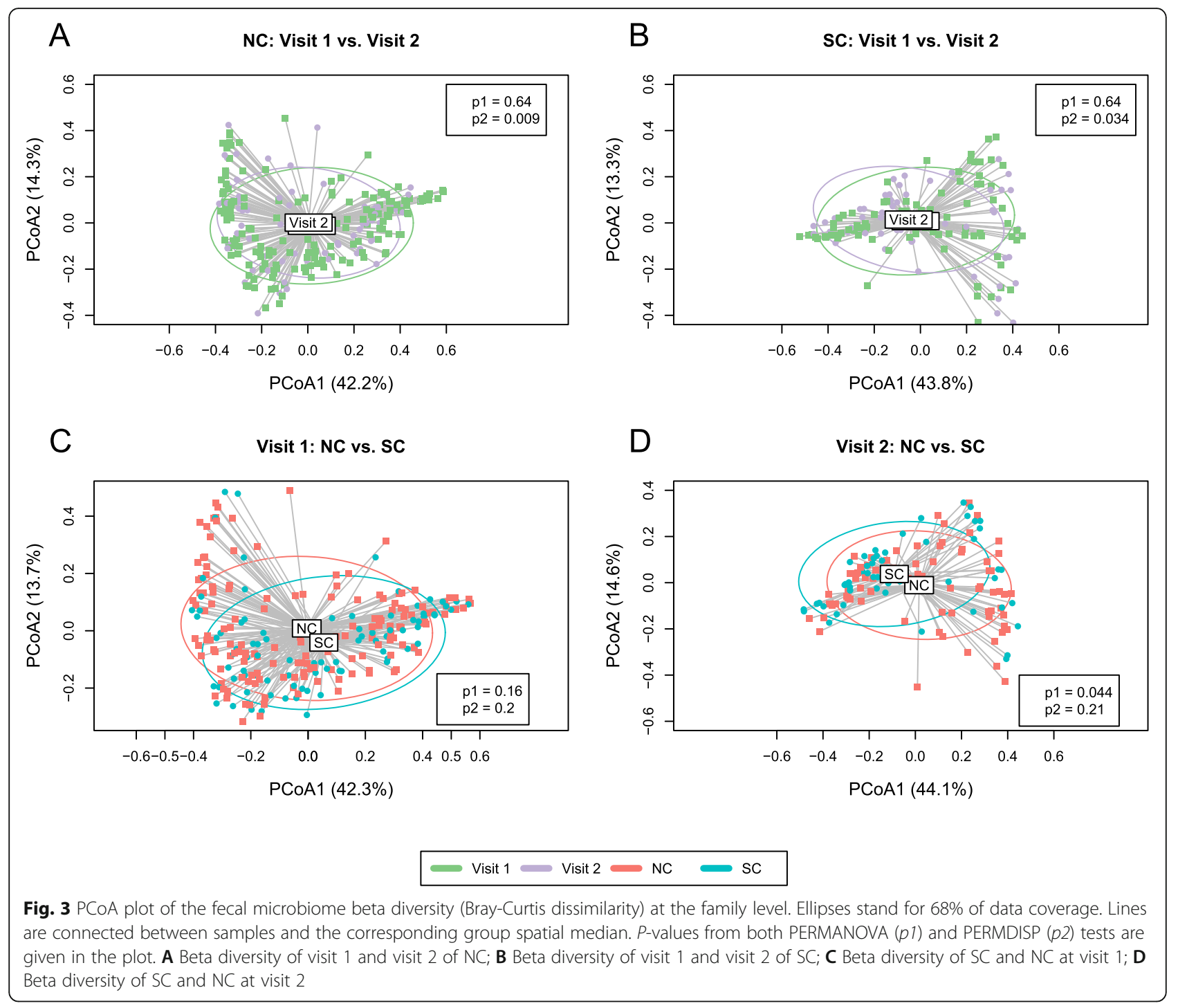

1.10, $p<0.05$ ) and 5 bacterial species (Butyricicoccus Pullicaecorum, Alistipes Onderdonkii, Bacteroides Caccae, Bacteroides Ovatus, and Alistipes Putredinis) were significantly lower in the mean abundance (LFC ranges from -1.14 to $-0.65, p<0.05)$ in $\mathrm{SC}$ compared to $\mathrm{NC}$ at visit 2 (Fig. 5B, Supplementary Table 3B). Compared to SC visit 1, 4 species (unknown, Parabacteroides distasonis, Blautia obeum, and Collinsella aerofaciens) were significantly lower in the mean abundance (LFC ranges from -0.92 to $-0.29, p<0.05)$ at SC visit 2 after HIV-1 seroconversion (Fig. 5C, Supplementary Table 3C). Since HIV-1 replicates rampantly in gut-associated lymphoid tissue and compromises the mucosal immune system during acute HIV-1 infection [36], the changes of bacterial taxa after HIV-1 infection in SC suggest a pathogenic impact of HIV-1 infection on the gut microbiome.

The bacterial composition of SCs was further analyzed according to the time to AIDS diagnosis. We discovered that Veillonellaceae was significantly higher $(p<0.05)$ in the mean abundance at visit 1 in SC with AIDS development within 5 years compared to the SC who were AIDS-free for more than 10 years (Fig. 4D, Supplementary Table 1D). At visit 2, Prevotellaceae was significantly higher $(p<0.05)$ in the mean abundance among the $\mathrm{SC}$ who developed AIDS within 5 years compared to the SC who were AIDS-free for more than 10 years. Additionally, Victivallaceae was only present in the SC with AIDS development within 5 years or 5-10 years compared to the SC who were AIDS-free for more than 10 years (Fig. 4E, Supplementary Table 1E). At the genus level, Methanosphaera was significantly higher $(p<0.05)$ and Eubacterium was significantly lower at visit 1 in SC with AIDS development within 5 years compared to the $\mathrm{SC}$ who were AIDS-free for more than 10 years (Supplementary Figure S5D, Supplementary Table 2D). At visit 2, the mean abundance of Prevotella was significantly 


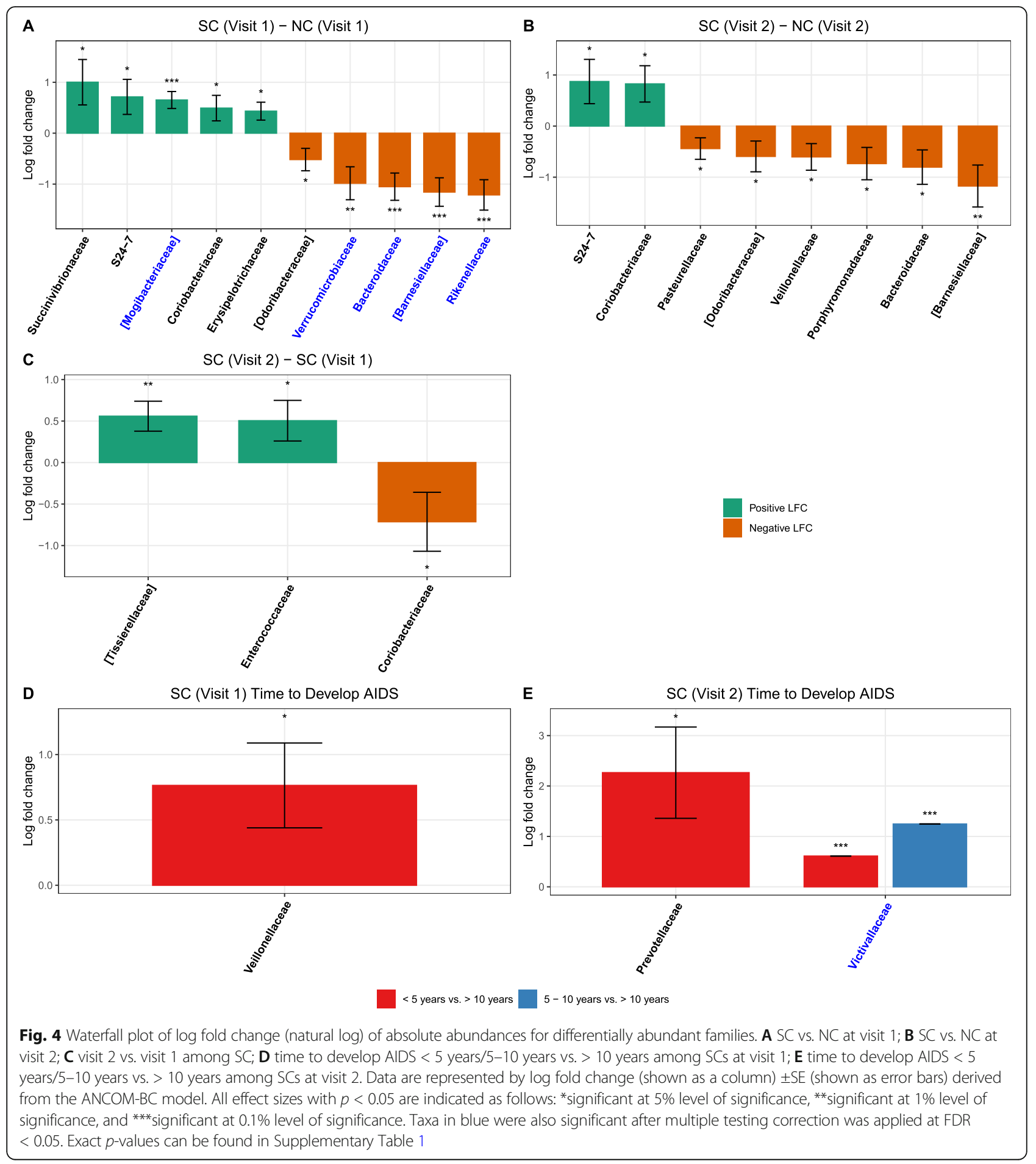

higher whereas Faecalibacterium, Anaerovibrio, and Paraprevotella had significantly lower mean abundance $(p<0.05$, Anaerovibrio was absent among AIDS rapid developers) in the SC who developed AIDS within 5 years compared to the SC who were AIDS-free for more than 10 years. Additionally, at visit 2, Mitsuokella, Butyrivibrio, Oribacterium, and Lachnospira were significantly higher and Blautia and Gemmiger were significantly lower in mean abundance $(p<0.05)$ in the SC with AIDS development within 5-10 years compared to the SC who were AIDS-free for more than 10 years (Supplementary Figure S5E, Supplementary Table 2E). At the species level, Dorea formicigenerans was significantly lower in mean abundance $(p<0.05)$ at visit 1 in SC with 


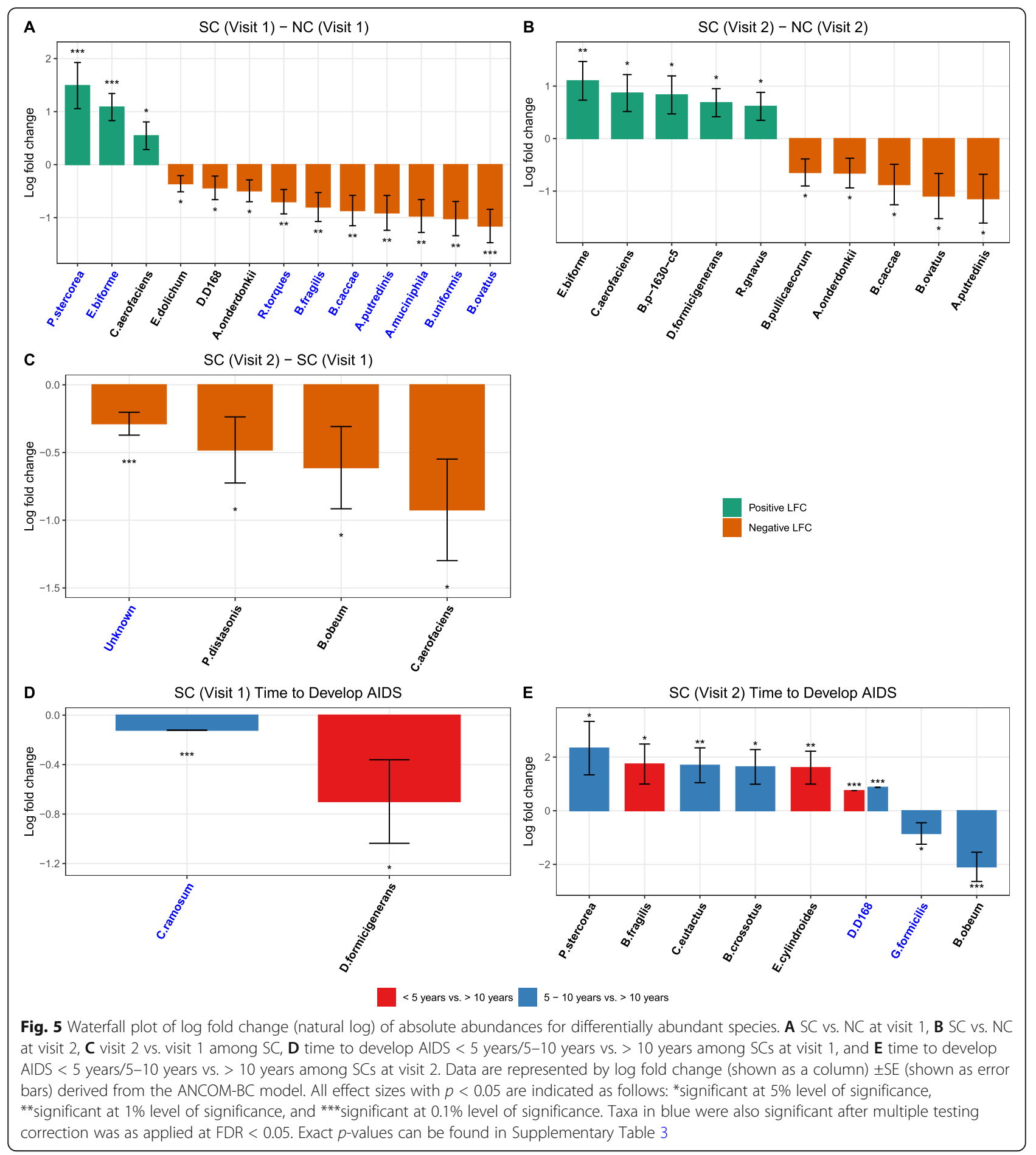

AIDS development within 5 years compared to the SC who were AIDS-free for more than 10 years. Additionally, Clostridium ramosum was absent in the SC with AIDS development within 5-10 years compared to the SC who were AIDS-free for more than 10 years (Fig. 5D, Supplementary Table 3D). At visit 2, Bacteroides fragilis, Eubacterium cylindroides, and Desulfovibrio D168 were significantly higher in mean abundance $(p<0.05$, where Desulfovibrio D168 is absent among AIDS long-term developers) in the SC who developed AIDS within 5 years compared to the SC who were AIDS-free for more than 10 years. Additionally, Prevotella stercorea, Coprococcus eutactus, Butyrivibrio crossotus, and Desulfovibrio D168 were significantly higher $(p<0.05$, Desulfovibrio D168 is 
absent among AIDS long-term developers) and Gemmiger formicilis and Blautia obeum were significantly lower in mean abundance $(p<0.05)$ at visit 2 in the SC with AIDS development within 5-10 years compared to the SC who were AIDS-free for more than 10 years (Fig. 5, Supplementary Table 3E).

\section{Fecal SCFA}

Gut microbes produce SCFA by fermenting dietary nondigestible carbohydrates. SCFA are important for intestinal and immune homeostasis. Unbalanced intestinal SCFA quantities play a role in HIV-1 pathogenesis [3739]. Therefore, four SCFA (acetate, butyrate, propionate, and valerate) were measured in the stool samples of both $\mathrm{NC}$ and SC at both visits. Although we did not find significant differences in the level of each individual SCFA between $\mathrm{NC}$ and $\mathrm{SC}$ at visit 1 or visit 2 (data not shown), analysis of SCFA level and $\mathrm{CD} 4^{+} / \mathrm{CD}^{+}$ratios (Fig. 6) showed there was a significant positive correlation between the propionate levels and $\mathrm{CD} 4^{+} / \mathrm{CD}^{+}$ratio at visit 1 of SC with $p=0.0022$ (Fig. 6A), and a trend of a positive correlation with $p=0.093$ at visit 2 in SC (Fig. $6 \mathrm{~B})$. Interestingly, there was no significant correlation between propionate levels and $\mathrm{CD} 4^{+} / \mathrm{CD}^{+}$ratio in $\mathrm{NC}$ in either visit (data not shown). Correlations between SCFA and plasma inflammatory cytokine levels at both visits were not statistically significant (data not shown). Correlations between SCFA levels and HIV-1 load at visit 2 in SC were not found to be significant (Supplementary Figure S6). Propionate, the conjugate base of propionic acid, is produced by gut bacteria Bacteroidetes, Firmicutes, and others. Propionate plays an important role in gut immune regulation. The positive association of propionate level and $\mathrm{CD} 4^{+} / \mathrm{CD}^{+}$ratio before HIV-1 infection could indicate propionate's role in HIV-1 acquisition due to the high level of $\mathrm{CD}_{4}^{+} \mathrm{T}$ cells.

\section{Plasma inflammatory cytokines}

Dynamic changes of pro-inflammatory cytokines in the peripheral blood play an important role in HIV-1

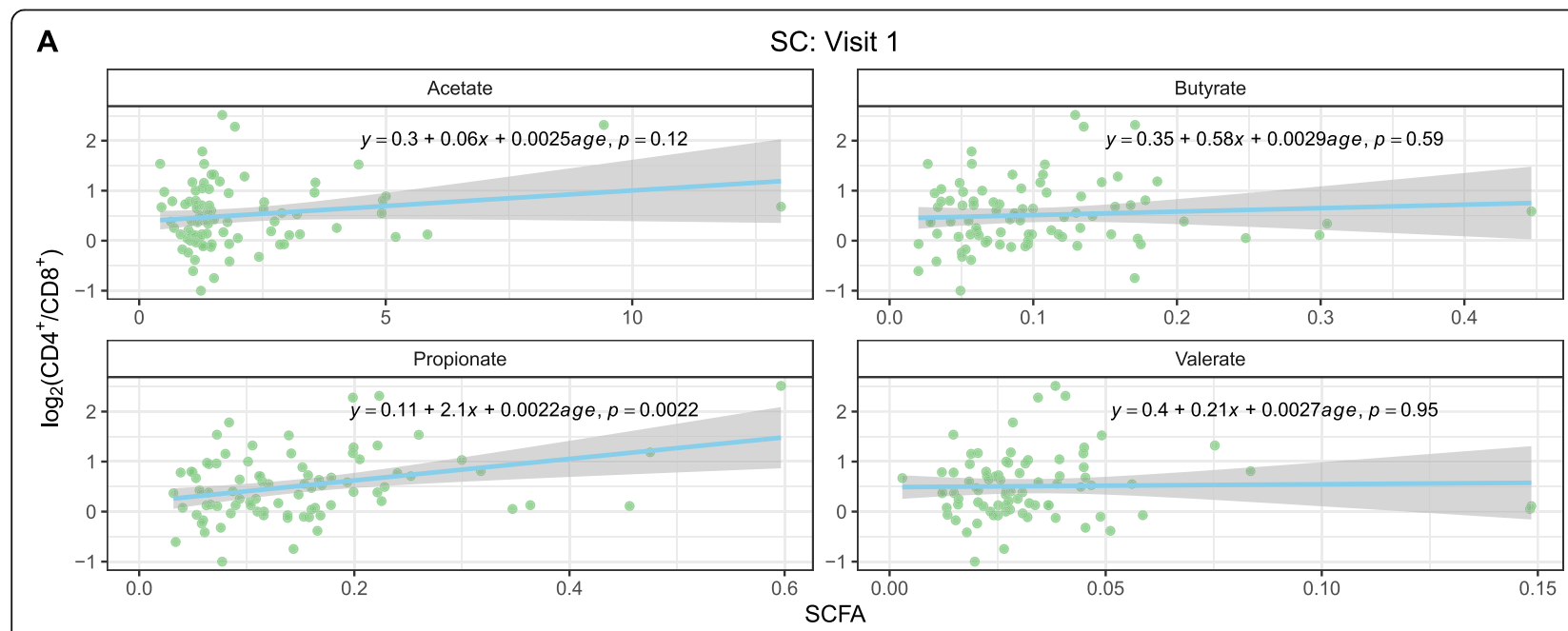

B

SC: Visit 2
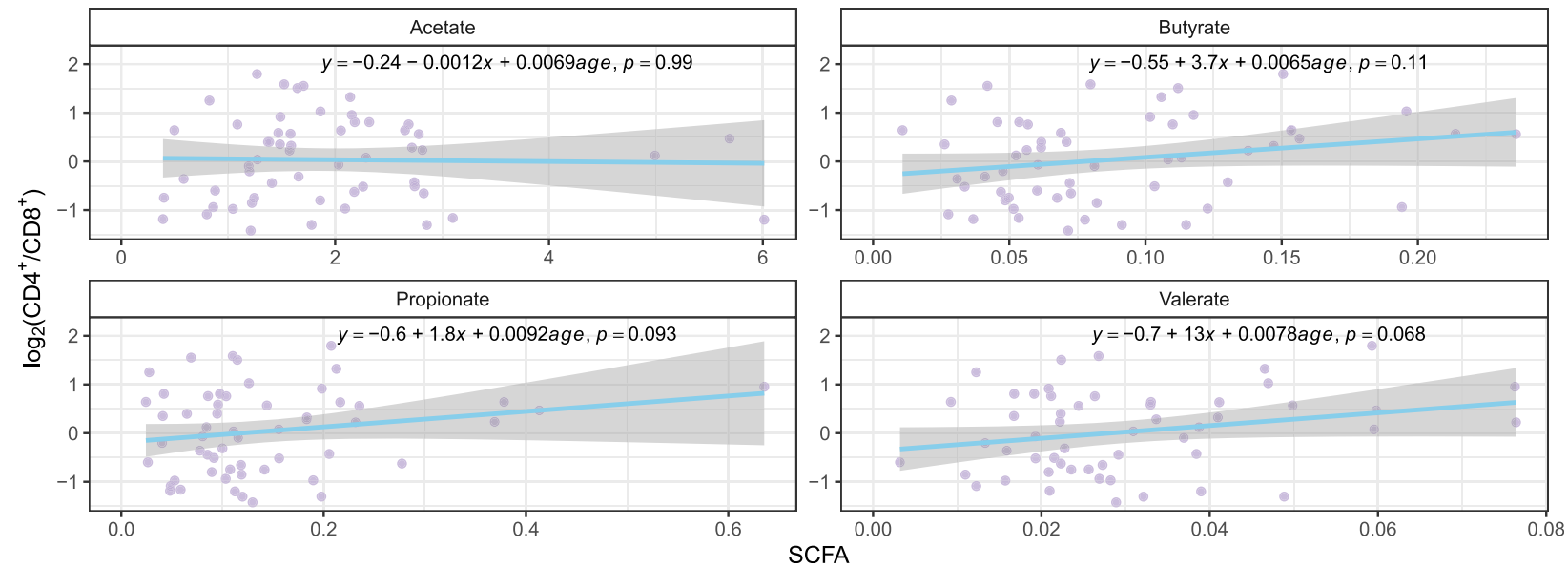

Fig. 6 Correlation of fecal SCFA levels and $\log _{2}$ ratio of the peripheral blood $C D 4^{+} / C D 8^{+}$at visit 1 (A) or visit 2 (B) of SC. SC visit 1: $N=87$, visit 2: $N=57$ 
acquisition and disease progression [40]. There was an effect of inflammation on the incidence of seroconversion to HIV-1, as the levels of plasma sCD14, sCD163, IL-6, and LBP were significantly higher prior to seroconversion at visit 1 in $\mathrm{SC}$ compared to visit 1 in $\mathrm{NC}$ (Fig. 7A). After seroconversion to HIV-1, levels of sCD163 and IP10 significantly increased in SC at visit 2 compared to pre-seroconversion visit 1 (Fig. 7B). None of the cytokine levels significantly correlated with $\mathrm{CD}^{+} /$ $\mathrm{CD}^{+}$ratio among $\mathrm{NC}$ at either visit (Supplementary Figure S7) or among SC at visit 1 (Fig. 8A), with the exception of IL- 6 and CRP at NC visit 1 . However, these positive correlations are likely driven by certain leverage points (observations for which the cytokine level is much higher than average). On the contrary, the levels of sCD14 and LBP at visit 2 among SC were positively correlated with $\mathrm{CD} 4^{+} / \mathrm{CD} 8^{+}$ratio $(p=0.0077$ and $p=0.0046$, respectively) (Fig. 8B). Levels of IP-10 and LBP at visit 2 among SC positively correlated with HIV-1 viral load with $p$-values $<0.01$ (Fig. 9).

\section{Correlation of microbiome before HIV-1 infection and HIV-1 seroconversion status and pathogenesis}

The role of the fecal microbiome in the HIV-1 susceptibility of MSM is not clear due to the challenges of collecting fecal materials before HIV-1 infection. In this study, we took the unique opportunity to analyze the fecal microbiome composition before HIV-1 infection and its implication in HIV-1 acquisition and pathogenesis post-HIV-1 infection in MSM.

\section{Microbiome alpha diversity at visit 1 and $C D 4^{+} / C D 8^{+}$at visit 2}

To explore the role of fecal bacterial within-sample diversity prior to HIV-1 infection in acute HIV-1 infection pathogenesis, Shannon diversity index at visit 1 was coanalyzed with peripheral blood $\mathrm{CD} 4^{+} / \mathrm{CD}^{+}$ratio at visit 2 in SC and NC. As shown in Supplementary Figure S8, although it was not statistically significant, fecal bacterial alpha diversity at visit 1 tended to be negatively correlated with $\mathrm{CD}^{+} / \mathrm{CD}^{+}$ratio at visit 2 in $\mathrm{SC}$, but not in NC. As the baseline diversity increases, it appears that the $\mathrm{CD}^{+} / \mathrm{CD}^{+}$ratio decreases dramatically post-HIV-1 infection for SC.

\section{Discussion}

The basic premise of our study is that the fecal microbiome has a major effect on the infection of MSM with HIV-1. This is based on known differences in enteric microbiota and systemic immune activation in non-HIV-1 infected MSM [19, 41], and in MSM with chronic HIV-1 infection [42-45]. Moreover, recent studies [19, 41, 46, 47] have shown that MSM have distinct microbiomes compared to MSW regardless of HIV-1 infection status.
In addition, $\mathrm{CD}^{+}{ }^{+} \mathrm{T}$ cells with high expression of HIV-1 co-receptor CCR5 have been detected in MSM rectosigmoid colon biopsies [48]. The impact of the fecal microbiome on HIV-1 infection is also evident in changes in uninfected individuals taking pre-exposure prophylaxis (PrEP) to prevent HIV-1 infection [49], and HIV-1infected individuals with ART [50-54] or without ART $[54,55]$. The non-human primate model also supports that a fecal microbiome with lower ratios of Bacteroides to Prevotella, and lower levels of Firmicutes are associated with mucosal transmission of high levels of simian-human immunodeficiency virus [56]. However, there is no information on whether these differences in the microbiome are linked to susceptibility to HIV-1 infection in MSM.

To address this issue, we applied unique data and biologic specimens from the earliest phase of the AIDS pandemic in MSM enrolled in the MACS to describe for the first time the dynamic changes of the gut microbiome, plasma inflammatory cytokines, and the peripheral blood $\mathrm{CD}^{+} / \mathrm{CD}^{+} \mathrm{T}$ cell ratio present several months before and after primary HIV-1 infection in MSM. We found that there were significant differences in the fecal microbiome compositions of SC and NC in our MSM cohort at their biannual study visit prior to HIV-1 infection. Our findings after HIV-1 infection in SC are in agreement with existing literature on high levels of Prevotella stercorea [13, 42, 46], Eubacterium biforme [13, 46] and low levels of Desulfovibrio D168 [19], Bacteroides fragilis [57], Bacteroides caccae [13, 46, 57], Akkermansia muciniphila [46, 58, 59], Bacteroides uniformis [13, 42, 46, 57], and Bacteroides ovatus [13, 46, 57] in PWH. Especially, significantly higher in the abundance of Prevotella stercorea $(p<0.01, \mathrm{BH}$ adjusted $p=0.01)$ and lower in the abundance of Bacteroides fragilis $(p<0.01, \mathrm{BH}$ adjusted $p=0.02)$, Bacteroides caccae $(p<0.01, \mathrm{BH}$ adjusted $p=0.02$ ), Bacteroides uniformis ( $\mathrm{p}<0.01, \mathrm{BH}$ adjusted $p=$ $0.02)$, and Bacteroides ovatus $(p<0.01, \mathrm{BH}$ adjusted $p=$ 0.01 ) were detected in SC before HIV-1 infection compared to NC. These data support that the pathogenic microbiome changes with high Prevotella and low Bacteroides occurred before HIV-1 infection in MSM. Although high levels of fecal Prevotella were reported in healthy Tanzania hunter-gatherers [60] and athletes [61], increases in fecal Prevotella and decreases in Bacteroides have also been found in various gut inflammatory conditions [62-64]. Our finding could indicate a proinflammatory condition in the GI tract in SC before acquiring HIV-1, in that P. stercorea species of the gut commensal bacterial genus Prevotella has proinflammatory effects, whereas several species of the genus Bacteroides have the anti-inflammatory function by promoting $\mathrm{T}$ regulatory cell reactivity [62, 65]. Pascal et al. [63] reported that fecal bacteria family Mogibacteriaceae along with other bacteria were 

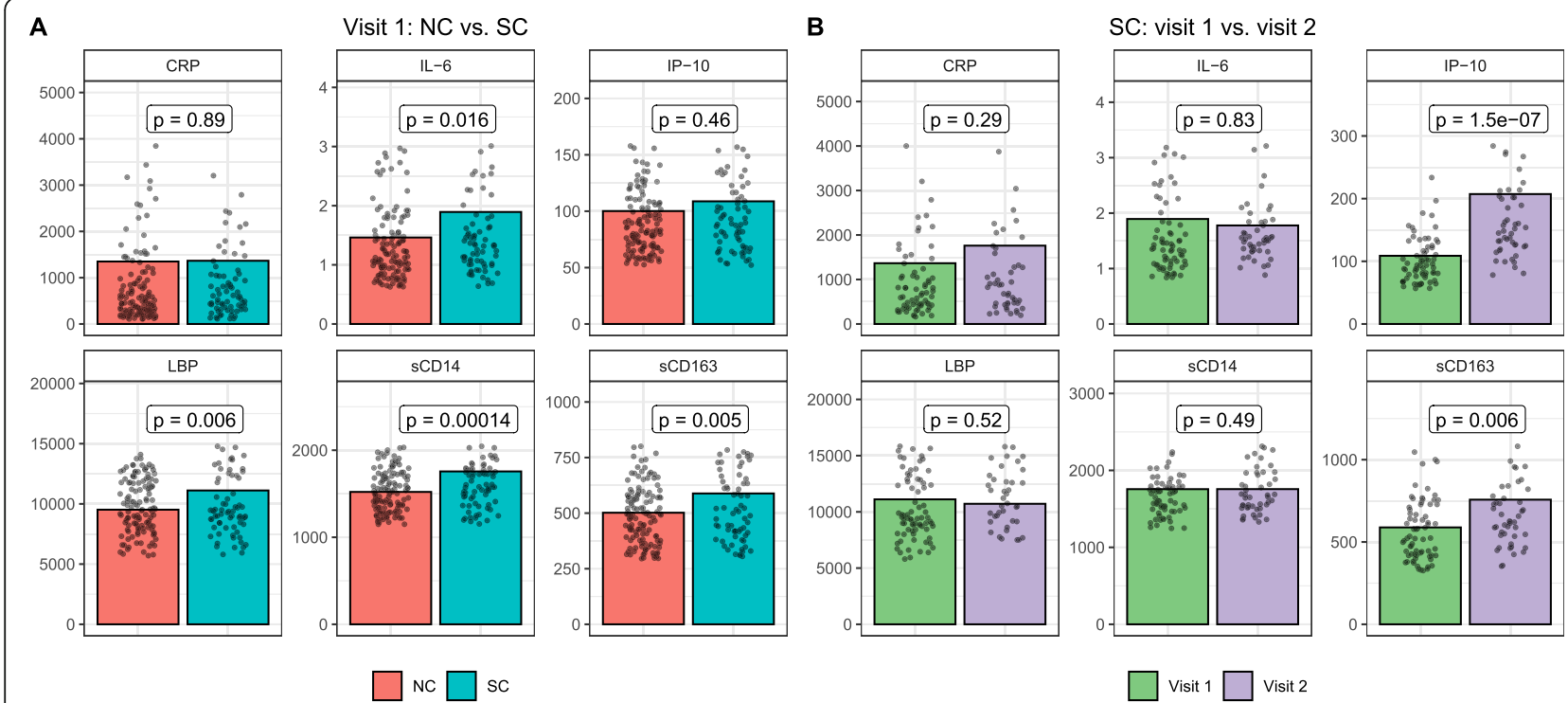

Fig. 7 A Relative abundances of peripheral blood inflammatory cytokines at visit 1 between NC ( $N=156)$ and SC ( $N=87)$. B Relative abundances of peripheral blood inflammatory cytokines between visit $1(N=87)$ and visit $2(N=57)$ of SC. The measurement for each cytokine is IL-6 ( $\mathrm{pg} / \mathrm{ml})$, sCD163 (ng/ml), IP-10 (pg/ml), CRP (ng/ml), LBP (ng/ml), and sCD14 (ng/ml). For visualization purposes, scatter points with values belonging to the first/last 10 percentile were not shown

significantly increased in inflammatory bowel disease (IBD) patients with proctitis. Similarly, the fecal bacteria family Erysipelotrichaceae is associated with HIV-1 infection [13, 66] and colorectal cancer [67, 68]. In our study participants, two potential pathogenic fecal bacteria, Mogibacteriaceae and Erysipelotrichaceae, were significantly increased in SC prior to HIV-1 infection compared to NC. In contrast, antiinflammatory and protective bacteria, Bacteroidaceae and Rikenellaceae, whose decrease is associated with HIV-1 infection and IBD [69-71], were significantly lower in SC prior to HIV-1 infection. These changes of microbiome composition in MSM before HIV-1 seroconversion could lead to greater inflammation in the GI tract and position them to be vulnerable to HIV-1 infection.

SCFA are fermentation products of gut microbiota from dietary fibers and a major factor in the maintenance of gut and immune homeostasis [72, 73]. Rau et al. [74] reported the association of higher fecal propionate and acetate levels with lower resting regulatory $\mathrm{T}$ cells and higher Th17/rTreg ratio in the peripheral blood in nonalcoholic fatty liver disease patients. In this study, we found a significant positive correlation between the levels of propionate and the peripheral blood $\mathrm{CD} 4^{+} / \mathrm{CD}^{+}$ratio before HIV-1 infection in SC. Although limited reports are available on propionate level changes in PWH [75] and in other diseases [76], whether the gut propionate level modulates the systemic immune system and influences HIV-1 susceptibility needs further investigation.
Immune activation after HIV-1 infection has been extensively reported [77-79]. However, there are very limited reports on immune turbulence surrounding HIV-1 infection, which is important for early therapeutic intervention. Breen et al. [80] reported that the significant changes of plasma cytokines 20 days before and 20 days after HIV-1 infection of 3 acute HIV-1-infected persons, showing high levels of plasma pro-inflammatory cytokines a few days post HIV-1 infection. In our current study, significantly high levels of pro-inflammatory cytokine sCD14, sCD163, IL-6, and LBP were detected in SC prior to HIV-1 infection compared to $\mathrm{NC}(p<$ $0.05)$. This systemic immune activation could be the consequence of microbial translocation caused by fecal microbiome dysbiosis, which was further supported by the significant increase in pro-inflammatory bacterial taxa (Prevotella stercorea) and a significant decrease in anti-inflammatory bacterial taxa (Bacteroides fragilis, Bacteroides caccae, Bacteroides uniformis, and Bacteroides ovatus) in SC prior to HIV-1 infection. These microbiome changes could increase local inflammation and mucosal membrane permeability, which may in turn lead to microbial translocation and systemic inflammation. In addition, this microbiome change could increase the number of local activated $\mathrm{CD} 4^{+} \mathrm{T}$ cells by immune activation and chemoattractant, which then serve as targets upon exposure to HIV-1 in the GI tract.

To our knowledge, our study is the only investigation linking the fecal microbiome present within several months before and after primary HIV-1 infection 


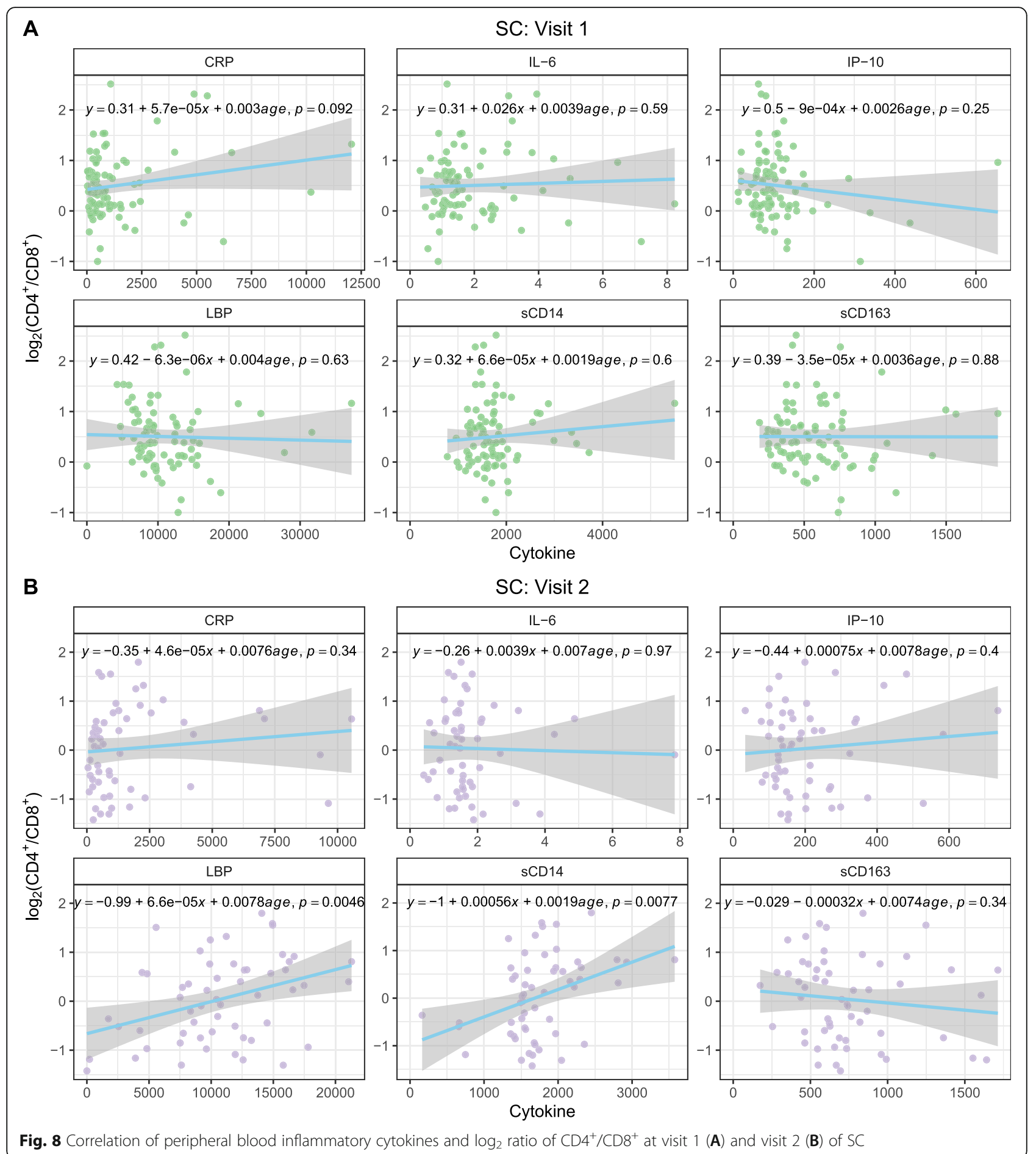

with the development of AIDS. After HIV-1 infection, we found that fecal Prevotellaceae, Victivallaceae, and Eubacterium cylindroides were significantly higher in SC who subsequently developed AIDS faster (within 5 years) compared to the SC who were AIDS-free for more than 10 years without ART. High levels of fecal Prevotellaceae and Eubacterium cylindroides are found in PWH [13, 46, 57], and fecal Victivallaceae are enriched in PWH on ART after nutritional intervention [81]. We recognize that given the relatively small number of PWH in our study for prediction modeling, caution is warranted in making 


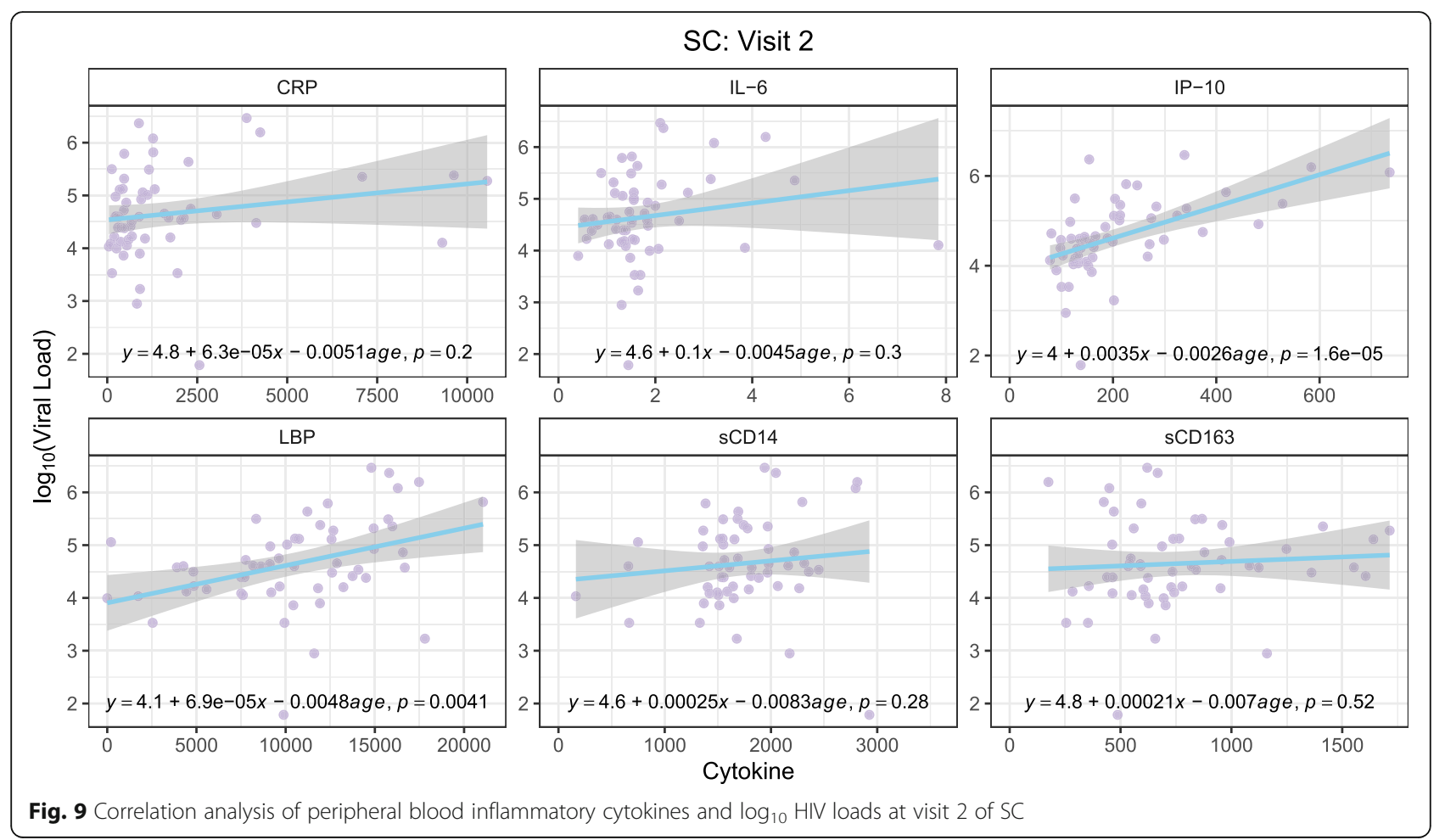

predictive conclusions on the role of the fecal microbiome in developing AIDS. Nevertheless, it is unlikely that our study can be reproduced due to the lack of stool specimens archived from other pre-ART, HIV/AIDS cohort studies and current treatment policies of rapidly initiating ART soon after primary HIV-1 infection.

The present results are pertinent to recent studies showing a distinct gut microbiome composition in MSM compared to MSW even in the absence of HIV-1 infection [18, 19, 41, 46]. Furthermore, Li et al. reported that mice receiving MSM stool samples showed increased activation of $\mathrm{CD} 4^{+} \mathrm{T}$ cells, and human gut-derived immune cells were more likely to be infected by HIV-1 after being exposed to MSM fecal bacteria in vitro [18]. Although all of our study participants were MSM, different homosexual behaviors of individual study participants might contribute to different levels of microbiome changes and local and systemic inflammation. Indeed, men who are exclusively receptive during anal intercourse are more likely to become infected with HIV-1 than exclusively insertive men $[22,82]$. It is possible that the microbiome differences observed in SC before HIV-1 infection are caused by different sexual behaviors of the study participants. Furthermore, since the fecal microbiome plays an important role in modulating the systemic immune system, the microbiome differences observed could be a driving factor for immune activation and susceptibility to HIV-1 infection. After HIV-1 infection, fecal alpha diversity of SC significantly decreased, which is consistent with a previous study by Noguera-Julian et al. [19]. Further investigation of the role of sexual history in the microbiome of our study participants is ongoing.

\section{Conclusions}

Our results support that pathogenic changes in the gut microbiome occurred in MSM several months prior to seroconversion to HIV-1. This was associated with increased inflammatory biomarkers in blood and increased risk for the development of AIDS.

\section{Abbreviations}

MSM: Men who have sex with men; HIV-1: Human immunodeficiency virus type 1; MACS: Multicenter AIDS Cohort Study; SC: HIV-1 seroconverters; NC: HIV-1 negative controls; SCFA: Short-chain fatty acids; ART: Antiretroviral drug therapy

\section{Supplementary Information}

The online version contains supplementary material available at https://doi. org/10.1186/s40168-021-01168-w.

Additional file 1. Supplementary figure S1. Sequence reads of bacterial 16 S rRNA V4 gene of SC and NC stool samples. Supplementary figure S2. Correlation analysis of fecal microbiome Shannon diversity index and $\log _{2}$ ratio of peripheral blood $\mathrm{CD}^{+} / \mathrm{CD}^{+}$at visit $1(\mathrm{~A})$ or visit $2(\mathrm{~B})$ of $\mathrm{SC}$ and NC. Supplementary figure S3. Correlation analysis of fecal microbiome Shannon diversity index and peripheral blood HIV loads at visit 2 of SC. Supplementary figure S4. The fecal microbiome 
compositions at phylum level of SC and NC at visit 1 and visit 2 obtained with $16 \mathrm{~S}$ rRNA gene sequencing. Supplementary figure S5. Waterfall plot of log fold change (natural log) of absolute abundances for differentially abundant genera. A: SC vs. NC at visit 1; B. SC vs. NC at visit 2; C. visit 2 vs. visit 1 among SC; D. Time to develop AIDS $<5$ years $/ 5-10$ years vs. $>$ 10 years among SCS at visit 1; E. Time to develop AIDS $<5$ years $/ 5-10$ years vs. $>10$ years among SCs at visit 2 . Data are represented by log fold change (shown as column) \pm SE (shown as error bars) derived from the ANCOM-BC model. All effect sizes with $p<0.05$ are indicated, ${ }^{*}$ significant at $5 \%$ level of significance; ${ }^{* *}$ significant at $1 \%$ level of significance; ***significant at $0.1 \%$ level of significance. Taxa in blue were also significant after multiple testing correction was applied at FDR $<0.05$. Exact $p$ values can be found in Supplementary Table 2. Supplementary figure S6. Correlation analysis of fecal SCFAs and $\log _{10}$ peripheral blood HIV loads at visit 2 of SC. Supplementary figure S7. Correlation of peripheral blood inflammatory cytokines and $\log _{2}$ ratio of $\mathrm{CD}^{+} / \mathrm{CD}^{+}$at visit 1 (A) and visit 2 (B) of NC. Supplementary figure S8. Correlation of fecal microbiome alpha diversity (Shannon diversity index) at visit 1 and $\log 2$ ratio of peripheral blood CD4+/CD8+ at visit 2 of SC and NC.

Additional file 2. Supplementary Table 1. The log fold change (LFC) of absolute abundances for differentially abundant families (A), (B), (C), (D), (E). *The taxon was declared to be significant since the absolute abundance in the reference group (Time to Develop AIDS > 10 Years) was zero. **NE: not evaluable. Supplementary Table 2. The log fold change (LFC) of absolute abundances for differentially abundant genera (A), (B), (C), (D), (E). *The taxon was declared to be significant since the absolute abundance in the group of interest (Time to Develop AIDS $<5$ Years) was zero. **NE: not evaluable. Supplementary Table 3. The log fold change (LFC) of absolute abundances for differentially abundant families $(A),(B),(C),(D),(E) .{ }^{*}$ The taxon was declared to be significant since the absolute abundance in the group of interest (Time to Develop AIDS 5 10 Years) was zero. ${ }^{* *}$ NE: not evaluable. ${ }^{* *}$ The taxon was declared to be significant since the absolute abundance in the reference group (Time to Develop AIDS > 10 Years) was zero.

\section{Acknowledgements}

Data in this manuscript were collected by the Multicenter AIDS Cohort Study (MACS), now the MACS/WIHS Combined Cohort Study (MWCCS). The contents of this publication are solely the responsibility of the authors and do not represent the official views of the National Institutes of Health $(\mathrm{NIH})$. MACS Principal Investigators: Johns Hopkins University, Baltimore MACS (Todd Brown and Joseph Margolick), U01-HL146201; Johns Hopkins University, MACS Data Analysis and Coordination Center (Gypsyamber D'Souza, Stephen Gange and Elizabeth Golub), U01HL146193; Northwestern University, Chicago MACS (Steven Wolinsky), U01-HL146240; University of California, Los Angeles MACS (Roger Detels and Matthew Mimiaga), U01HL146333; University of Pittsburgh MACS (Jeremy Martinson and Charles Rinaldo), U01-HL146208. The authors gratefully acknowledge the contributions of the study participants and dedication of the staff at the MWCCS sites.

The authors thank Jay Hayes and Kathy Kulka from the Pittsburgh MACS for laboratory technical support, Jeffrey Toth from the Pittsburgh MACS data center and Andrea Stronski from the Center for the Analysis of MACS data for data support, Dr. Bernard J. Macatangay for providing laboratory facility support for the cyokine testing, Dr. John Mellors and Dr. Steven Wolinsky for the helpful comments, and especially the original, 1984-1985 participants of the Multicenter AIDS Cohort Study without whom this study would not be possible.

\section{Authors' contributions}

YC, MC, and AF performed fecal $16 \mathrm{~S}$ rRNA sequencing experiments, YC and VRS performed the cytokine measurements, HM and BM provided the data management and technical support, and $\mathrm{HL}$ and SP analyzed the $16 \mathrm{~S}$ rRNA sequencing data. YC, HL, JM, AM, HM, SP, and CRR formulated the study, interpreted the data, and contributed to writing the manuscript. The authors read and approved the final manuscript.

\section{Funding}

This work was supported by N01-Al-32513, U01-HL-146208, and NIHS10OD023402 from the National Institutes of Health.

\section{Availability of data and materials}

The datasets generated and/or analyzed during the current study are available in the GitHub repository (https://github.com/FrederickHuangLin/ Gut-Microbiome-and-HIV-Infection).

\section{Declarations}

Ethics approval and consent to participate

The study was conducted with institutional review board approvals from the University of Pittsburgh, Northwestern University, University of California, Los Angeles, and Johns Hopkins University.

\section{Consent for publication}

Not applicable.

\section{Competing interests}

The authors declare that they have no competing interests.

\section{Author details}

${ }^{3}$ Current address: Biostatistics and Bioinformatics Branch, Eunice Kennedy Shriver National Institute of Child Health and Human Development (NICHD), $\mathrm{NIH}$, Bethesda, MD, USA. 'Department of Infectious Diseases and Microbiology, University of Pittsburgh Graduate School of Public Health, Pittsburgh, PA, USA. ${ }^{2}$ Department of Biostatistics, University of Pittsburgh Graduate School of Public Health, Pittsburgh, PA, USA. ${ }^{4}$ Present address: Wilmot Cancer Center, University of Rochester Medical Center, Rochester, NY, USA. ${ }^{5}$ Division of Pulmonary, Allergy and Critical Care Medicine, Department of Medicine, University of Pittsburgh School of Medicine, Pittsburgh, PA, USA. ${ }^{6}$ Department of Epidemiology, The Johns Hopkins Bloomberg School of Public Health, Baltimore, MD, USA. ${ }^{7}$ Department of Epidemiology, Fielding School of Public Health, University of California at Los Angeles, Los Angeles, CA, USA. ${ }^{8}$ Department of Molecular Microbiology and Immunology, The Johns Hopkins Bloomberg School of Public Health, Baltimore, MD, USA.

Received: 2 June 2021 Accepted: 14 September 2021

Published online: 09 December 2021

\section{References}

1. Ahluwalia B, Magnusson MK, Ohman L. Mucosal immune system of the gastrointestinal tract: maintaining balance between the good and the bad. Scand J Gastroenterol. 2017;52(11):1185-93.

2. Kurashima $Y$, Kiyono H. Mucosal ecological network of epithelium and immune cells for gut homeostasis and tissue healing. Annu Rev Immunol. 2017;35:119-47.

3. Sanz Y, De Palma G. Gut microbiota and probiotics in modulation of epithelium and gut-associated lymphoid tissue function. Int Rev Immunol. 2009:28(6):397-413.

4. Lujan JA, Rugeles MT, Taborda NA. Contribution of the microbiota to intestinal homeostasis and its role in the pathogenesis of HIV-1 infection. Curr HIV Res. 2019;17(1):13-25.

5. Dillon SM, Frank DN, Wilson CC. The gut microbiome and HIV-1 pathogenesis: a two-way street. AIDS. 2016;30(18):2737-51.

6. Bandera A, De Benedetto I, Bozzi G, Gori A. Altered gut microbiome composition in HIV infection: causes, effects and potential intervention. Curr Opin HIV AIDS. 2018;13(1):73-80.

7. Wang C, Li Q, Ren J. Microbiota-immune interaction in the pathogenesis of gut-derived infection. Front Immunol. 2019;10:1873.

8. Liu J, Williams B, Frank D, Dillon SM, Wilson CC, Landay AL, Inside out: HIV the gut microbiome, and the mucosal immune system. J Immunol. 2017; 198(2):605-14

9. McDermott AJ, Huffnagle GB. The microbiome and regulation of mucosal immunity. Immunology. 2014;142(1):24-31.

10. Tincati C, Douek DC, Marchetti G. Gut barrier structure, mucosal immunity and intestinal microbiota in the pathogenesis and treatment of HIV infection. AIDS Res Ther. 2016:13:19.

11. Zevin AS, McKinnon L, Burgener A, Klatt NR. Microbial translocation and microbiome dysbiosis in HIV-associated immune activation. Curr Opin HIV AIDS. 2016;11(2):182-90.

12. Monaco CL, Gootenberg DB, Zhao G, Handley SA, Ghebremichael MS, Lim $E S$, et al. Altered virome and bacterial microbiome in human 
immunodeficiency virus-associated acquired immunodeficiency syndrome. Cell Host Microbe. 2016;19(3):311-22.

13. Lozupone CA, Li M, Campbell TB, Flores SC, Linderman D, Gebert MJ, et al. Alterations in the gut microbiota associated with HIV-1 infection. Cell Host Microbe. 2013;14(3):329-39.

14. Shukla S, Kumari S, Bal SK, Monaco DC, Ribeiro SP, Sekaly RP, et al. "Go", "No Go," or "Where to Go"; does microbiota dictate T cell exhaustion, programming, and HIV persistence? Curr Opin HIV AIDS. 2021.

15. Gori A, Tincati C, Rizzardini G, Torti C, Quirino T, Haarman M, et al. Early impairment of gut function and gut flora supporting a role for alteration of gastrointestinal mucosa in human immunodeficiency virus pathogenesis. J Clin Microbiol. 2008;46(2):757-8.

16. Rocafort M, Noguera-Julian M, Rivera J, Pastor L, Guillen Y, Langhorst J, et al. Evolution of the gut microbiome following acute HIV-1 infection. Microbiome. 2019;7(1):73

17. Moore RD. Epidemiology of HIV infection in the United States: implications for linkage to care. Clin Infect Dis. 2011;52(Suppl 2):S208-13.

18. Li SX, Sen S, Schneider JM, Xiong KN, Nusbacher NM, Moreno-Huizar N, et al. Gut microbiota from high-risk men who have sex with men drive immune activation in gnotobiotic mice and in vitro HIV infection. PLoS Pathog. 2019;15(4):e1007611.

19. Noguera-Julian M, Rocafort M, Guillen Y, Rivera J, Casadella M, Nowak P, et al. Gut microbiota linked to sexual preference and hiv infection. EBioMedicine. 2016;5:135-46.

20. Tuddenham S, Koay WL, Sears C. HIV, sexual orientation, and gut microbiome interactions. Dig Dis Sci. 2020;65(3):800-17.

21. Kingsley LA, Detels R, Kaslow R, Polk BF, Rinaldo CR Jr, Chmiel J, et al. Risk factors for seroconversion to human immunodeficiency virus among male homosexuals. Results from the Multicenter AIDS Cohort Study. Lancet. 1987; 1(8529):345-9.

22. Baggaley RF, White RG, Boily MC. HIV transmission risk through anal intercourse: systematic review, meta-analysis and implications for HIV prevention. Int J Epidemiol. 2010;39(4):1048-63.

23. Kaslow RA, Ostrow DG, Detels R, Phair JP, Polk BF, Rinaldo CR Jr. The Multicenter AIDS Cohort Study: rationale, organization, and selected characteristics of the participants. Am J Epidemiol. 1987;126(2):310-8.

24. Lyles RH, Munoz A, Yamashita TE, Bazmi H, Detels R, Rinaldo CR, et al. Natural history of human immunodeficiency virus type 1 viremia after seroconversion and proximal to AIDS in a large cohort of homosexual men. Multicenter AIDS Cohort Study. J Infect Dis. 2000;181(3):872-80

25. Jacobson LP, Kirby AJ, Polk S, Phair JP, Besley DR, Saah AJ, et al. Changes in survival after acquired immunodeficiency syndrome (AIDS): 1984-1991. Am J Epidemiol. 1993;138(11):952-64.

26. 1993 revised classification system for HIV infection and expanded surveillance case definition for AIDS among adolescents and adults. MMWR Recomm Rep. 1992:41(RR-17):1-19.

27. Caporaso JG, Lauber CL, Walters WA, Berg-Lyons D, Lozupone CA, Turnbaugh PJ, et al. Global patterns of 165 rRNA diversity at a depth of millions of sequences per sample. Proc Natl Acad Sci U S A. 2011;108(Suppl 1):4516-22.

28. Han J, Lin K, Sequeira C, Borchers CH. An isotope-labeled chemical derivatization method for the quantitation of short-chain fatty acids in human feces by liquid chromatography-tandem mass spectrometry. Anal Chim Acta. 2015;854:86-94.

29. Lin H, Peddada SD. Analysis of compositions of microbiomes with bias correction. Nat Commun. 2020;11(1):3514.

30. Kaul A, Mandal S, Davidov O, Peddada SD. Analysis of microbiome data in the presence of excess zeros. Front Microbiol. 2017:8:2114.

31. Benjamini $Y$, Hochberg $Y$. Controlling the false discovery rate: a practical and powerful approach to multiple testing. J R Stat Soc Ser B Methodol. 57: 289-300.

32. Shetty SA, Hugenholtz F, Lahti L, Smidt H, de Vos WM. Intestinal microbiome landscaping: insight in community assemblage and implications for microbia modulation strategies. FEMS Microbiol Rev. 2017;41(2):182-99.

33. Weiss S, Xu ZZ, Peddada S, Amir A, Bittinger K, Gonzalez A, et al Normalization and microbial differential abundance strategies depend upon data characteristics. Microbiome. 2017:5(1):27.

34. Mandal S, Van Treuren W, White RA, Eggesbo M, Knight R, Peddada SD. Analysis of composition of microbiomes: a novel method for studying microbial composition. Microb Ecol Health Dis. 2015;26:27663.
35. McBride JA, Striker R. Imbalance in the game of T cells: what can the CD4/ CD8 T-cell ratio tell us about HIV and health? PLoS Pathog. 2017;13(11): e1006624.

36. Brenchley JM, Douek DC. HIV infection and the gastrointestinal immune system. Mucosal Immunol. 2008;1(1):23-30.

37. Dillon SM, Kibbie J, Lee EJ, Guo K, Santiago ML, Austin GL, et al. Low abundance of colonic butyrate-producing bacteria in HIV infection is associated with microbial translocation and immune activation. AIDS. 2017; 31(4):511-21.

38. Serrano-Villar S, Vazquez-Castellanos JF, Vallejo A, Latorre A, Sainz T, Ferrando-Martinez $\mathrm{S}$, et al. The effects of prebiotics on microbial dysbiosis, butyrate production and immunity in HIV-infected subjects. Mucosal Immunol. 2017;10(5):1279-93.

39. Gonzalez-Hernandez LA, Del Rocio Ruiz-Briseno M, Sanchez-Reyes K, Alvarez-Zavala M, Vega-Magana N, Lopez-Iniguez A, et al. Correction to: Alterations in bacterial communities, SCFA and biomarkers in an elderly HIVpositive and HIV-negative population in western Mexico. BMC Infect Dis. 2020;20(1):764

40. Breen EC. Pro- and anti-inflammatory cytokines in human immunodeficiency virus infection and acquired immunodeficiency syndrome. Pharmacol Ther. 2002;95(3):295-304.

41. Kelley CF, Kraft CS, de Man TJ, Duphare C, Lee HW, Yang J, et al. The rectal mucosa and condomless receptive anal intercourse in HIV-negative MSM: implications for HIV transmission and prevention. Mucosal Immunol. 2017; 10(4):996-1007.

42. Dillon SM, Lee EJ, Kotter CV, Austin GL, Dong Z, Hecht DK, et al. An altered intestinal mucosal microbiome in HIV-1 infection is associated with mucosal and systemic immune activation and endotoxemia. Mucosal Immunol. 2014 7(4):983-94.

43. Mutlu EA, Keshavarzian A, Losurdo J, Swanson G, Siewe B, Forsyth C, et al. A compositional look at the human gastrointestinal microbiome and immune activation parameters in HIV infected subjects. PLoS Pathog. 2014;10(2): e1003829.

44. Vazquez-Castellanos JF, Serrano-Villar S, Latorre A, Artacho A, Ferrus ML, Madrid N, et al. Altered metabolism of gut microbiota contributes to chronic immune activation in HIV-infected individuals. Mucosal Immunol. 2015;8(4):760-72.

45. Lu W, Feng $Y$, Jing $F$, Han $Y$, Lyu N, Liu F, et al. Association between gut microbiota and CD4 recovery in HIV-1 infected patients. Front Microbiol. 2018;9:1451.

46. Armstrong AJS, Shaffer M, Nusbacher NM, Griesmer C, Fiorillo S, Schneider $\mathrm{JM}$, et al. An exploration of Prevotella-rich microbiomes in HIV and men who have sex with men. Microbiome. 2018;6(1):198.

47. Vujkovic-Cvijin I, Sortino O, Verheij E, Sklar J, Wit FW, Kootstra NA, et al. HIVassociated gut dysbiosis is independent of sexual practice and correlates with noncommunicable diseases. Nat Commun. 2020;11(1):2448.

48. Coleman SL, Neff CP, Li SX, Armstrong AJS, Schneider JM, Sen S, et al. Can gut microbiota of men who have sex with men influence HIV transmission? Gut Microbes. 2020;11(3):610-9.

49. Perler BK, Reinhart EM, Montgomery M, Maynard M, Shapiro JM, Belenky P, et al. Evaluation of the microbiome in men taking pre-exposure prophylaxis for HIV prevention. AIDS Behav. 2021;25(7):2005-13.

50. Nganou-Makamdop K, Talla A, Sharma AA, Darko S, Ransier A, Laboune F, et al. Translocated microbiome composition determines immunological outcome in treated HIV infection. Cell. 2021:184(15):3899-3914 e3816.

51. Bai X, Narayanan A, Nowak P, Ray S, Neogi U, Sonnerborg A. Wholegenome metagenomic analysis of the gut microbiome in hiv-1-infected individuals on antiretroviral therapy. Front Microbiol. 2021;12:667718.

52. Ancona G, Merlini E, Tincati C, Barassi A, Calcagno A, Augello M, et al. Longterm suppressive CART is not sufficient to restore intestinal permeability and gut microbiota compositional changes. Front Immunol. 2021;12:639291.

53. Dillon SM, Abdo MH, Wilson MP, Liu J, Jankowski CM, Robertson CE, et al. A unique gut microbiome-physical function axis exists in older people with HIV: an exploratory study. AIDS Res Hum Retrovir. 2021;37(7):542-50.

54. Armstrong AJS, Quinn K, Fouquier J, Li SX, Schneider JM, Nusbacher NM, et al. Systems analysis of gut microbiome influence on metabolic disease in HIV-positive and high-risk populations. mSystems. 2021;6(3).

55. do Nascimento WM, Machiavelli A, Ferreira LGE, Cruz Silveira L, de Azevedo SSD, Bello G, et al. Gut microbiome profiles and associated metabolic pathways in HIV-infected treatment-naive patients. Cells. 2021:10(2). 
56. Sui Y, Dzutsev A, Venzon D, Frey B, Thovarai V, Trinchieri G, et al. Influence of gut microbiome on mucosal immune activation and SHIV viral transmission in naive macaques. Mucosal Immunol. 2018;11(4):1219-29.

57. Cook RR, Fulcher JA, Tobin NH, Li F, Lee D, Javanbakht M, et al. Effects of HIV viremia on the gastrointestinal microbiome of young MSM. AIDS. 2019; 33(5):793-804.

58. Neff CP, Krueger O, Xiong K, Arif S, Nusbacher N, Schneider JM, et al. Fecal microbiota composition drives immune activation in hiv-infected individuals. EBioMedicine. 2018;30:192-202.

59. Dubourg G, Lagier JC, Hue S, Surenaud M, Bachar D, Robert C, et al. Gut microbiota associated with HIV infection is significantly enriched in bacteria tolerant to oxygen. BMJ Open Gastroenterol. 2016;3(1):e000080.

60. Schnorr SL, Candela M, Rampelli S, Centanni M, Consolandi C, Basaglia G, et al. Gut microbiome of the Hadza hunter-gatherers. Nat Commun. 2014;5: 3654.

61. Mohr AE, Jager R, Carpenter KC, Kerksick CM, Purpura M, Townsend JR, et al. The athletic gut microbiota. J Int Soc Sports Nutr. 2020;17(1):24.

62. Larsen JM. The immune response to Prevotella bacteria in chronic inflammatory disease. Immunology. 2017;151(4):363-74.

63. Pascal V, Pozuelo M, Borruel N, Casellas F, Campos D, Santiago A, et al. A microbial signature for Crohn's disease. Gut. 2017;66(5):813-22.

64. Dinh DM, Volpe GE, Duffalo C, Bhalchandra S, Tai AK, Kane AV, et al. Intestinal microbiota, microbial translocation, and systemic inflammation in chronic HIV infection. J Infect Dis. 2015;211(1):19-27.

65. Jiang F, Meng D, Weng M, Zhu W, Wu W, Kasper D, et al. The symbiotic bacterial surface factor polysaccharide $A$ on Bacteroides fragilis inhibits IL1 beta-induced inflammation in human fetal enterocytes via toll receptors 2 and 4. PLoS One. 2017;12(3):e0172738.

66. Lozupone CA, Rhodes ME, Neff CP, Fontenot AP, Campbell TB, Palmer BE. HIV-induced alteration in gut microbiota: driving factors, consequences, and effects of antiretroviral therapy. Gut Microbes. 2014;5(4):562-70.

67. Chen W, Liu F, Ling Z, Tong X, Xiang C. Human intestinal lumen and mucosa-associated microbiota in patients with colorectal cancer. PLoS One. 2012;7(6):e39743.

68. Kaakoush NO. Insights into the role of Erysipelotrichaceae in the human host. Front Cell Infect Microbiol. 2015;5:84.

69. Mazmanian SK, Round JL, Kasper DL. A microbial symbiosis factor prevents intestinal inflammatory disease. Nature. 2008;453(7195):620-5.

70. Round JL, Mazmanian SK. Inducible Foxp3+ regulatory T-cell development by a commensal bacterium of the intestinal microbiota. Proc Natl Acad Sci U S A. 2010;107(27):12204-9.

71. Vatanen T, Kostic AD, d'Hennezel E, Siljander H, Franzosa EA, Yassour M, et al. Variation in microbiome LPS immunogenicity contributes to autoimmunity in humans. Cell. 2016;165(4):842-53.

72. Tan J, McKenzie C, Potamitis M, Thorburn AN, Mackay CR, Macia L. The role of short-chain fatty acids in health and disease. Adv Immunol. 2014;121:91119.

73. Li M, van Esch B, Wagenaar GTM, Garssen J, Folkerts G, Henricks PAJ. Proand anti-inflammatory effects of short chain fatty acids on immune and endothelial cells. Eur J Pharmacol. 2018;831:52-9.

74. Rau M, Rehman A, Dittrich M, Groen AK, Hermanns HM, Seyfried F, et al. Fecal SCFAs and SCFA-producing bacteria in gut microbiome of human NAFLD as a putative link to systemic T-cell activation and advanced disease. United European Gastroenterol J. 2018;6(10):1496-507.

75. Gonzalez-Hernandez LA, Ruiz-Briseno MDR, Sanchez-Reyes K, Alvarez-Zavala M, Vega-Magana N, Lopez-Iniguez A, et al. Alterations in bacterial communities, SCFA and biomarkers in an elderly HIV-positive and HIVnegative population in western Mexico. BMC Infect Dis. 2019;19(1):234.

76. Bindels LB, Porporato P, Dewulf EM, Verrax J, Neyrinck AM, Martin JC, et al. Gut microbiota-derived propionate reduces cancer cell proliferation in the liver. Br J Cancer. 2012;107(8):1337-44.

77. Wada NI, Bream JH, Martinez-Maza O, Macatangay B, Galvin SR, Margolick JB, et al. Inflammatory biomarkers and mortality risk among hiv-suppressed men: a multisite prospective cohort study. Clin Infect Dis. 2016;63(7):984-90.

78. Connolly NC, Riddler SA, Rinaldo CR. Proinflammatory cytokines in HIV disease-a review and rationale for new therapeutic approaches. AIDS Rev. 2005;7(3):168-80.

79. Brenchley JM, Price DA, Schacker TW, Asher TE, Silvestri G, Rao S, et al. Microbial translocation is a cause of systemic immune activation in chronic HIV infection. Nat Med. 2006;12(12):1365-71.
80. Breen EC, Reynolds SM, Cox C, Jacobson LP, Magpantay L, Mulder CB, et al. Multisite comparison of high-sensitivity multiplex cytokine assays. Clin Vaccine Immunol. 2011;18(8):1229-42.

81. Serrano-Villar S, de Lagarde M, Vazquez-Castellanos J, Vallejo A, Bernadino I, Madrid $\mathrm{N}$, et al. Effects of immunonutrition in advanced human immunodeficiency virus disease: a randomized placebo-controlled clinical trial (promaltia study). Clin Infect Dis. 2019;68(1):120-30.

82. Patel P, Borkowf CB, Brooks JT, Lasry A, Lansky A, Mermin J. Estimating peract HIV transmission risk: a systematic review. AIDS. 2014;28(10):1509-19.

\section{Publisher's Note}

Springer Nature remains neutral with regard to jurisdictional claims in published maps and institutional affiliations.

\section{Ready to submit your research? Choose BMC and benefit from:}

- fast, convenient online submission

- thorough peer review by experienced researchers in your field

- rapid publication on acceptance

- support for research data, including large and complex data types

- gold Open Access which fosters wider collaboration and increased citations

- maximum visibility for your research: over $100 \mathrm{M}$ website views per year

At BMC, research is always in progress.

Learn more biomedcentral.com/submissions 